\title{
GENERAL-RELATIVISTIC SIMULATIONS OF THREE-DIMENSIONAL CORE-COLLAPSE SUPERNOVAE
}

\author{
Christian D. Ott ${ }^{1,2,10}$, Ernazar Abdikamalov ${ }^{1}$, Philipp Mösta $^{1}$, Roland Haas ${ }^{1}$, Steve Drasco $^{1,3}$, \\ Evan P. O’Connor ${ }^{1,4}$, Christian Reisswig ${ }^{1,11}$, Casey A. Meakin ${ }^{5,6}$, and Erik Schnetter ${ }^{7,8,9}$ \\ ${ }^{1}$ TAPIR, Mailcode 350-17, California Institute of Technology, Pasadena, CA 91125, USA; cott@ tapir.caltech.edu \\ ${ }^{2}$ Kavli Institute for the Physics and Mathematics of the Universe (Kavli IPMU), The University of Tokyo, Kashiwa, Japan \\ ${ }^{3}$ Grinnell College, Grinnell, IA, USA \\ ${ }^{4}$ Canadian Institute for Theoretical Astrophysics, University of Toronto, Toronto, ON, Canada \\ 5 Theoretical Division, Los Alamos National Laboratory, Los Alamos, NM, USA \\ ${ }^{6}$ Steward Observatory, University of Arizona, Tucson, AZ, USA \\ ${ }^{7}$ Perimeter Institute for Theoretical Physics, Waterloo, ON, Canada \\ ${ }^{8}$ Department of Physics, University of Guelph, Guelph, ON, Canada \\ ${ }^{9}$ Center for Computation \& Technology, Louisiana State University, Baton Rouge, LA, USA \\ Received 2012 November 20; accepted 2013 March 19; published 2013 April 19
}

\begin{abstract}
We study the three-dimensional (3D) hydrodynamics of the post-core-bounce phase of the collapse of a $27 M_{\odot}$ star and pay special attention to the development of the standing accretion shock instability (SASI) and neutrino-driven convection. To this end, we perform 3D general-relativistic simulations with a three-species neutrino leakage scheme. The leakage scheme captures the essential aspects of neutrino cooling, heating, and lepton number exchange as predicted by radiation-hydrodynamics simulations. The $27 M_{\odot}$ progenitor was studied in 2D by Müller et al., who observed strong growth of the SASI while neutrino-driven convection was suppressed. In our 3D simulations, neutrino-driven convection grows from numerical perturbations imposed by our Cartesian grid. It becomes the dominant instability and leads to large-scale non-oscillatory deformations of the shock front. These will result in strongly aspherical explosions without the need for large-scale SASI shock oscillations. Low- $\ell$-mode SASI oscillations are present in our models, but saturate at small amplitudes that decrease with increasing neutrino heating and vigor of convection. Our results, in agreement with simpler 3D Newtonian simulations, suggest that once neutrino-driven convection is started, it is likely to become the dominant instability in 3D. Whether it is the primary instability after bounce will ultimately depend on the physical seed perturbations present in the cores of massive stars. The gravitational wave signal, which we extract and analyze for the first time from 3D general-relativistic models, will serve as an observational probe of the postbounce dynamics and, in combination with neutrinos, may allow us to determine the primary hydrodynamic instability.
\end{abstract}

Key words: gravitation - gravitational waves - hydrodynamics - neutrinos - supernovae: general

Online-only material: color figures

\section{INTRODUCTION}

Baade \& Zwicky (1934) inaugurated core-collapse supernova theory with their seminal prediction that "a super-nova represents the transition of an ordinary star into a neutron star." The very basics of this theory, summarized authoritatively by Bethe (1990), were confirmed by the observation of neutrinos from SN 1987A (Hirata et al. 1987; Bionta et al. 1987): the electrondegenerate core of a massive star (with mass $M \sim 8-130 M_{\odot}$ at zero-age main sequence), once having reached its effective Chandrasekhar mass, becomes radially unstable. Collapse ensues and, once fully dynamic, separates the core into the homologous, subsonically contracting inner core and the outer core, which is supersonically infalling. When the inner core reaches nuclear density, the nuclear force, which is repulsive at short distances, leads to a stiffening of the nuclear equation of state (EOS). The dramatically increased pressure support stabilizes the inner core, which overshoots its new equilibrium, then rebounds into the still infalling outer core. This core bounce launches a hydrodynamic shock wave, which, endowed with the kinetic energy of the inner core, plows into the outer core. Its progression is, however, soon muffled by energy losses to the

\footnotetext{
${ }^{10}$ Alfred P. Sloan Research Fellow.

${ }^{11}$ NASA Einstein Fellow.
}

dissociation of heavy nuclei and to electron capture neutrinos that are created and stream out from now optically-thin regions behind the shock. The hydrodynamic shock thus succumbs to the extreme ram pressure of the outer core and turns into a stalled accretion shock. In the commonly accepted picture of the neutrino mechanism (Wilson 1985; Bethe \& Wilson 1985; Bethe 1990; Janka et al. 2007), the shock is revived by the deposition of neutrino energy in a layer of net neutrino heating (the gain layer) below the shock. In an alternative scenario, requiring very rapid progenitor rotation and efficient magnetic field amplification, a magnetorotational explosion may occur (e.g., Burrows et al. 2007b and references therein). In order to leave behind a slowly cooling neutron star and not a black hole, shock revival must occur within a few hundred milliseconds of bounce (O'Connor \& Ott 2011; Ugliano et al. 2012).

While the general picture of core-collapse supernova theory may be well established, details of the explosion mechanism, its dependence on precollapse conditions and input physics, and its neutrino and gravitational wave $(\mathrm{GW})$ signals $^{12}$ remain to be determined by detailed first-principles numerical simulations.

\footnotetext{
12 Both neutrinos and gravitational waves may be direct probes of progenitor properties, supernova dynamics, and of the explosion mechanism. See, e.g., Ott (2009), Lund et al. (2010), Lund et al. (2012), Dasgupta et al. (2010), Brandt et al. (2011), Logue et al. (2012), and O’Connor \& Ott (2013).
} 
In the case of the neutrino mechanism, modern sphericallysymmetric (1D) simulations with full Boltzmann neutrino transport have shown that neutrino heating alone fails to drive an explosion in all but the lowest-mass massive stars (Liebendörfer et al. 2001; Rampp \& Janka 2002; Thompson et al. 2003; Sumiyoshi et al. 2005; Kitaura et al. 2006; Hüdepohl et al. 2010; Burrows et al. 2007a). Spherical symmetry, however, is a poor approximation to the situation after core bounce, even if the initial conditions are nearly spherically symmetric. The weakening shock leaves behind a negative entropy gradient, which is expected to lead to convective instability within milliseconds after bounce (prompt convection). Somewhat later, neutrino heating establishes a negative entropy gradient in the gain region, leading to neutrino-driven convection. Strong deleptonization near the neutrinosphere (where the neutrino optical depth $\tau_{v} \sim 1$; located at the edge of the protoneutron star at $\sim 10^{11}-10^{12} \mathrm{~g} \mathrm{~cm}^{-3}$ ) establishes a negative lepton gradient, driving protoneutron star convection.

The first full axisymmetric (2D) simulations (Herant et al. 1994; Burrows et al. 1995; Janka \& Müller 1995, 1996; Fryer \& Heger 2000) showed that 2D neutrino-driven convection could increase the efficacy of the neutrino mechanism by increasing the residence time of accreted material in the region of net neutrino heating and leading to high-entropy turbulent flow that aids shock expansion.

A new instability, the standing accretion shock instability (SASI), was discovered by Blondin et al. (2003), who carried out idealized 2D simulations of an accretion shock using an analytic EOS, neutrino cooling, but no neutrino heating. In 2D, the SASI leads to large-scale, low-order (in terms of spherical harmonics, $\ell=\{1,2\})$ deformations of the shock front that vary in time in a predominantly $\ell=1$ sloshing-type motion up and down the symmetry axis. These aspherical motions lead to larger average shock radii, increase the dwell time of material in the gain region, may lead to secondary shocks, and are thus generally aiding the explosion mechanism (Ohnishi et al. 2006; Scheck et al. 2006; Murphy \& Burrows 2008; Ott et al. 2008; Marek \& Janka 2009; Müller et al. 2012b). In 3D, nonaxisymmetric modes $(m=\{-\ell, \ldots, 0, \ldots, \ell\})$ are excited as well, leading to more complex dynamics and smaller saturation amplitudes for individual modes (Iwakami et al. 2008). In some 3D simulations, in particular in those that include some initial rotation, a strong spiral mode $(\ell=1, m= \pm 1)$, capable of redistributing angular momentum, has been observed (Blondin \& Mezzacappa 2007; Iwakami et al. 2008, 2009; Fernández 2010; Wongwathanarat et al. 2010; Rantsiou et al. 2011).

Perturbation theory and carefully controlled numerical experiments suggest that the SASI is driven by an advective-acoustic cycle in which entropy and vorticity perturbations are advected from the shock front to the edge of the protoneutron star. There they trigger the emission of acoustic perturbations that travel upstream in the subsonic flow of the postshock region and amplify perturbations in the shock front, thus creating a feedback cycle that injects power preferentially into low-order modes (see Foglizzo 2002; Foglizzo et al. 2006, 2007; Ohnishi et al. 2006; Yamasaki \& Yamada 2007; Fernández \& Thompson 2009a, 2009b; Scheck et al. 2008; Guilet \& Foglizzo 2012 and references therein). The saturation of the SASI has been proposed to occur via parasitic Rayleigh-Taylor and/or Kelvin-Helmholtz instabilities that operate on the entropy gradients and vorticity generated by the SASI (Guilet et al. 2010).

In a real core-collapse supernova, neutrino-driven convection and SASI overlap in space and may grow at the same time. In the linear regime, in which seed perturbations are minute, they can be clearly separated: SASI's fastest growing mode is $\ell=1$, while convective eddies will grow with horizontal wavelengths a few times of the entropy scale height, giving $\ell \sim 7-8$ in the postbounce supernova context (based on estimates of Foglizzo et al. 2006; Herant et al. 1992; see also Chandrasekhar 1961). In convection, however, all modes are unstable and will eventually grow to nonlinear amplitudes if convection is able to develop at all.

How convection and SASI interact in the nonlinear regime, which of them becomes the dominant instability, how this may depend on the dimensionality ( $2 \mathrm{D}$ versus $3 \mathrm{D}$ ), and the ramifications of all this for the explosion mechanism are open questions that are currently under much debate.

Foglizzo et al. (2006) argued, based on linear theory, that in the absence of large (i.e., nonlinear) perturbations the development of neutrino-driven convection may be suppressed if slowly developing eddies are advected out of the convectively unstable region before they can grow significantly. In this scenario, SASI would be the primary instability. This was also found in the idealized simulations of Ohnishi et al. (2006), who studied the $2 \mathrm{D}$ evolution of an artificially set up accretion shock with a constant accretion rate and analytic neutrino cooling, heating, and deleptonization functions. Scheck et al. (2008) performed 2D energy-averaged (gray) neutrino radiation-hydrodynamics postbounce simulations of a $15 M_{\odot}$ progenitor star in a carefully controlled setting to study the development of the SASI. They, too, confirmed the result of Foglizzo et al. (2006) and showed that if sufficiently large $(\gtrsim 1 \%)$ perturbations from sphericity are present in the upstream flow, neutrino-driven convection becomes the primary and dominant instability.

If linearly-growing convection is suppressed by high advection velocities in the gain region, then one would expect a dependence of the relative importance of SASI and convection on the postbounce accretion rate and, hence, on the progenitor star. This was convincingly confirmed by the recent work of Müller et al. (2012a), who carried out full first-principles 2D general-relativistic (GR) multi-energy radiation-hydrodynamics postbounce simulations of a $8.1 M_{\odot}$ low-metallicity star with a small core and low postbounce accretion rate and of a $27 M_{\odot}$ star of solar metallicity with a large core and high accretion rate. In agreement with the prediction of Foglizzo et al. (2006), they found strong convection and absent SASI in the $8.1 M_{\odot}$ star and strong SASI and nearly absent convection in the $27 M_{\odot}$ progenitor. In both cases, explosions developed within $\sim 200 \mathrm{~ms}$ of bounce.

In a different line of research targeted at understanding the dependence of the neutrino mechanism on dimensionality, Nordhaus et al. (2010) carried out 1D, 2D, and 3D Newtonian collapse simulations of a $15 M_{\odot}$ progenitor. They used the simple analytic heating and cooling prescription introduced by Murphy \& Burrows (2008; hereafter the MB08 "light-bulb" scheme) on the basis of the work of Janka (2001). Their 3D simulations did not show a dominant $\ell=1$ oscillatory SASI mode observed in 2D (Scheck et al. 2006; Ohnishi et al. 2006; Murphy \& Burrows 2008). Using the critical luminosity versus accretion rate approach of Burrows \& Goshy (1993), they reported that in 3D explosions could be obtained at $\sim 15 \%-25 \%$ and $\sim 40 \%-50 \%$ lower neutrino luminosities than in $2 \mathrm{D}$ and $1 \mathrm{D}$, respectively.

The Nordhaus et al. (2010) 3D versus 2D result was not confirmed by Hanke et al. (2012). These authors performed Newtonian simulations with neutrino approximations very similar to 
the MB08 light bulb, but used a different 3D hydrodynamics code. They did not find clear evidence that 3D effects facilitate the development of an explosion to a greater degree than the non-radial motions due to SASI and convection in 2D. However, in agreement with Nordhaus et al. (2010), they did not find large-scale oscillatory low-order modes in their 3D simulations. They hypothesized that this may be less of a 3D effect than an effect of the rather simple treatment of neutrino heating and cooling by Nordhaus et al. (2010). The arguably greatest limitation of the MB08 light-bulb scheme is its inability to track the contraction of the protoneutron star, leading to too low advection velocities in the gain region, thus artificially favoring neutrino-driven convection over the SASI. The results of Takiwaki et al. (2012), whose Newtonian 3D simulations used a multi-energy approximate neutrino transport scheme, appear supportive of this assertion. However, these simulations were carried out with very low resolution and the low-order modes appear to be clearly oscillatory only at early times.

Using the same MB08 light-bulb approximation for neutrinos and an updated version of the Nordhaus et al. (2010) code, Burrows et al. (2012), Murphy et al. (2012), and Dolence et al. (2013) performed and analyzed another set of 2D and 3D simulations to investigate the roles of SASI and convection in the postbounce evolution of a $15 M_{\odot}$ progenitor. Comparing $2 \mathrm{D}$ and $3 \mathrm{D}$ results for the evolution of low-order fluid mode amplitudes, Burrows et al. (2012) showed that at the same MB08 driving luminosity, oscillatory mode amplitudes are much smaller in $3 \mathrm{D}$ than in $2 \mathrm{D}$. In models that develop an explosion a non-oscillatory $\ell=1$ dipole asphericity grows already in the early postbounce evolution. Furthermore, they showed that the oscillatory $\ell=1$ modes observed in 2D-and generally associated with the SASI-occur even in the case of a high light-bulb driving luminosity, in which neutrinodriven convection is the dominant instability. They argued that in successful explosions by the neutrino mechanism, neutrinodriven convection should be the dominant instability. However, for the reasons put forth by Hanke et al. (2012) and Müller et al. (2012a) and discussed in the above, the predictive power of these light-bulb simulations may be limited.

Ultimately, high-resolution 3D energy-dependent GR neutrino radiation-hydrodynamics simulations will be needed for final answers regarding the explosion mechanisms and the role of the various instabilities involved. Such simulations are computationally extremely challenging and current attempts are forced to use low spatial resolution (Takiwaki et al. 2012; Kuroda et al. 2012), the gray approximation (Wongwathanarat et al. 2010; Müller et al. 2012c; Kuroda et al. 2012), and/or employ an artificial inner boundary, cutting out the protoneutron star core (Wongwathanarat et al. 2010; Müller et al. 2012c).

In this paper, we present results from $3 \mathrm{D}$ hydrodynamic postbounce supernova calculations that attempt to strike a balance between the computationally cheap, but possibly too simplistic light-bulb approximation and true 3D radiation-hydrodynamics simulations, which cannot yet be performed without at least partially debilitating limitations. Our simulations use the Zelmani core-collapse simulation package (Ott et al. 2012) and are fully GR. We make no symmetry assumptions and use no artificial inner boundary. We employ a novel computational setup with a multi-block approach that provides curvilinear grid blocks to track the collapse of the outer core and Cartesian adaptive mesh refinement (AMR) grids covering the central region, including the protoneutron star and the entire shock. We treat neutrinos in the postbounce phase with an energy-averaged three-species neutrino leakage scheme with neutrino heating. The only free parameter of this scheme is a scaling factor in the chargedcurrent energy deposition rate. As we shall demonstrate, the leakage scheme captures the essential aspects of neutrino cooling, neutrino heating, and lepton number exchange.

We apply Zelmani to the collapse and postbounce evolution of the $27 M_{\odot}$ progenitor star that was considered by Müller et al. (2012a) and shown to be highly susceptible to the SASI in their fully self-consistent 2D GR simulations. Müller et al. (2012a) find a SASI-aided explosion that develops within $\sim 150-200 \mathrm{~ms}$ after bounce, making this progenitor ideal for studying the SASI in computationally expensive high-resolution 3D simulations. We carry out four simulations of the $27 M_{\odot}$ progenitor, varying the strength of neutrino heating. We evolve these four models from the onset of collapse to $\sim 150-190 \mathrm{~ms}$ after bounce at an effective angular resolution of 0.85 at a radius of $100 \mathrm{~km}$. The linear resolution at this radius is $\sim 1.5 \mathrm{~km}$. The maximum resolution covering the protoneutron star core is $\sim 370 \mathrm{~m}$.

We find that neutrino-driven convection is able to grow from the numerical seed perturbations imposed by our Cartesian AMR approach. It becomes the dominant instability in the postbounce dynamics of all of our models. In the case of strong neutrino heating, convection, which is initially manifest as small-scale cells of rising hotter and sinking cooler material, develops into large blobs of high-entropy material. These push out the shock and lead to large-scale non-oscillatory shock deformations. We also observe growth of oscillatory low- $(\ell, m)$ deformations associated with the SASI. However, these saturate at small amplitudes that decrease further with increasing strength of neutrino heating and vigor of convection. The SASI remains sub-dominant at all times in our simulations. Our results suggest that if neutrino-driven convection is able to grow in $3 \mathrm{D}$ - which will generally depend on the postbounce accretion rate and on the seed perturbations present in the flow (Scheck et al. 2008) - it will dominate the postbounce flow. This is consistent with the results obtained by Burrows et al. (2012) with the simpler light-bulb approach. We extract the GW signals generated by accelerated quadrupole mass motions in our models and find that the strongest component of the signal comes from the initial burst of convection, which grows on the negative entropy gradient left behind by the stalling shock.

This paper is structured as follows. In Section 2, we describe Zelmani and give details on grid setup, EOS, the leakage/heating scheme, and the progenitor model. In Section 3, we present the results of our simulations. First, in Section 3.1, we give an overview of the overall postbounce evolution of our models. We then discuss in detail the postbounce configurations resulting from our leakage/heating scheme (Section 3.2), the development of neutrino-driven convection and SASI (Section 3.3), various criteria for neutrino-driven explosions (Section 3.4), and the GW signals extracted from our models (Section 3.5). We summarize our findings and conclude in Section 4.

\section{METHODS AND INITIAL CONDITIONS}

We carry out our 3D GR simulations with the Zelmani core-collapse simulation package. Zelmani is based on the open-source Einstein Toolkit ${ }^{13}$ (Löffler et al. 2012), for numerical relativity and relativistic computational astrophysics. It builds upon the Carpet AMR driver (Schnetter et al. 2004) and the Llama multi-block system (Pollney et al. 2011; Reisswig

\footnotetext{
13 http://www.einsteintoolkit.org
} 
et al. 2013) within the Cactus Computational Toolkit (Goodale et al. 2003).

\subsection{Spacetime Evolution and Hydrodynamics}

We evolve the full Einstein equations without approximations in a $3+1$ decomposition as a Cauchy initial boundary value problem (see, e.g., Baumgarte \& Shapiro 2010), using the conformal-traceless BSSN formulation (Baumgarte \& Shapiro 1999; Shibata \& Nakamura 1995). A $1+\log$ slicing condition (Alcubierre et al. 2000) controls the evolution of the lapse function $\alpha$, and a modified $\Gamma$-driver condition (Alcubierre et al. 2003 ) is used for the evolution of the coordinate shift vector $\beta^{i}$. The BSSN equations and the gauge conditions are implemented in the module CTGamma using fourth-order accurate finite differencing. Implementation details are given in Pollney et al. (2011) and Reisswig et al. (2013). We note that in its present form, our evolution system is limited to 3D simulations. An extension to 2D along the lines of Baumgarte et al. (2013) may be possible in future work.

We use a flux-conservative formulation of the GR Euler equations, implemented in the GR hydrodynamics module GRHydro, which is part of the Einstein Toolkit (Löffler et al. 2012). GRHydro is an enhanced derivative of the Whisky (Baiotti et al. 2005) and GR-Astro/MAHC (Font et al. 2000) codes. It is based on a finite-volume high-resolution shock-capturing scheme and works with general finite-temperature microphysical EOS. We employ the enhanced piecewise-parabolic method for reconstruction of state variables at cell interfaces (McCorquodale \& Colella 2011; Reisswig et al. 2013) and subsequently solve approximate Riemann problems to compute intercell fluxes with the HLLE solver (Einfeldt 1988). Details are given in Reisswig et al. (2013).

Both spacetime evolution and GR hydrodynamics are discretized in a semi-discrete fashion and coupled with the Method of Lines (Hyman 1976) using a multi-rate Runge-Kutta integrator (Reisswig et al. 2013), providing fourth-order and secondorder accuracy in time for spacetime and GR hydrodynamics, respectively. The time step is limited by the speed of light and we use a constant Courant-Friedrichs-Lewy factor of 0.4.

\subsection{Multi-block Infrastructure, Adaptive Mesh Refinement, and Grid Setup}

We employ the multi-block infrastructure Llama (Pollney et al. 2011; Reisswig et al. 2013), which allows us to cover the computational domain using a set of overlapping curvilinear grid blocks that are logically Cartesian but physically curvilinear (so-called "inflated cubes"), adapted to the overall spherical topology of the collapse problem. We employ a set of such curvilinear blocks to track the collapse of the outer core, while the interior domain containing the protoneutron star, the postshock region, and the shock itself is covered by an adaptively refined Cartesian mesh (see Figure 1 for a schematic view).

The spherical inflated-cube multi-block system discretizes one spherical shell via six angular grid blocks designed such that one angular coordinate direction always coincides at interblock boundaries. This allows us to use efficient fourth-order one-dimensional interpolation to update ghost zone information between neighboring blocks (Thornburg 2004). Furthermore, this particular multi-block system offers an almost uniform distribution of points across the sphere (i.e., without clustering of points at the poles), thus avoiding distortions and pathologies associated with standard spherical-polar grids.

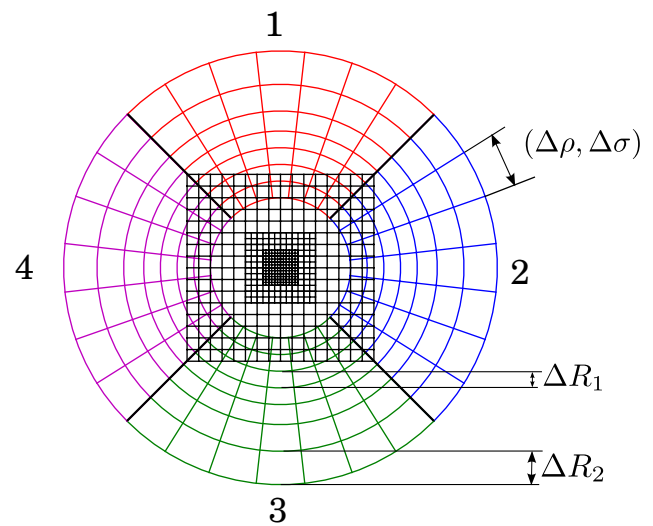

Figure 1. Schematic view of a slice through our 3D multi-block grid. Six physically curvilinear (four are shown), logically Cartesian inflated-cube grids with constant angular, varying radial resolution surround a central Cartesian region with five AMR levels (not all shown). The third finest level is adjusted to always encompass the entire postshock region.

(A color version of this figure is available in the online journal.)

The adaptively refined central Cartesian block is based on cell-centered and flux-conservative mesh-refinement techniques, provided by the open-source AMR driver Carpet (Schnetter et al. 2004; Reisswig et al. 2013). AMR is implemented with subcycling in time, following the approach of Berger \& Oliger (1984). We make use of refluxing, which correctly adjusts fluxes at mesh refinement boundaries after the AMR restriction operation (Reisswig et al. 2013). This ensures that mass, momentum, and energy fluxes are exactly conserved, even in the presence of strong shocks and other discontinuities. To update zones at AMR boundaries and to initialize new grid points after regridding, we make use of fourth-order prolongation for the spacetime curvature variables, and second-order essentially non-oscillatory prolongation for the matter variables. As detailed in Reisswig et al. (2013), spacetime variables are restricted from fine onto coarse grids using a third-order polynomial, while matter variables are restricted via cell averaging.

All simulations are carried out with the same general grid setup. In the central region, we use five nested Cartesian grids with a factor of two in resolution between each of them. The finest grid has a linear cell size $d x=0.37 \mathrm{~km}$ and extends out to $17.7 \mathrm{~km}$. The second finest grid has a cell size of $d x=0.74 \mathrm{~km}$ and extends to $59 \mathrm{~km}$, while the third grid has $d x=1.48 \mathrm{~km}$ and is set up to adaptively track the shock, ensuring that shock itself and the turbulent flow in the gain layer behind the shock are always resolved with no worse resolution than $d x=1.48 \mathrm{~km}$. For a shock radius of $100 \mathrm{~km}$, this corresponds to an effective angular resolution $d x / R$ of $\sim 0.85$. There are two additional coarser grids with $d x=2.95 \mathrm{~km}$ and $d x=5.9 \mathrm{~km}$ in the Cartesian region, which extends to $532 \mathrm{~km}$, where it overlaps with the outer spherical cube grid. The latter's radial cell size $d r$ at its inner boundary is the same as the $d x$ of the coarsest Cartesian grid it overlaps with. $d r$ is held constant out to a radius of $\sim 3000 \mathrm{~km}$ and then smoothly reduced to $d r=189 \mathrm{~km}$ at the outer boundary at $\sim 15000 \mathrm{~km}$. Each of the six cubedsphere blocks has 31 angular zones each in angle $\sigma$ and $\rho$. This corresponds to an effective cell size of $\sim 2.9$.

We start our simulations at the onset of collapse with only the coarsest of the Cartesian AMR grids active and progressively activate the finer grids when the central density in the collapsing core reaches $3.2 \times 10^{11} \mathrm{~g} \mathrm{~cm}^{-3}, 1.3 \times 10^{12} \mathrm{~g} \mathrm{~cm}^{-3}$, $5.1 \times 10^{12} \mathrm{~g} \mathrm{~cm}^{-3}$, and $2.0 \times 10^{13} \mathrm{~g} \mathrm{~cm}^{-3}$, respectively. 


\subsection{Equation of State}

We employ a tabulated version of the finite-temperature nuclear EOS by Lattimer \& Swesty (1991). This EOS is based on the compressible liquid-drop model with a nuclear symmetry energy of $29.3 \mathrm{MeV}$. We use its variant with a nuclear compression modulus $K_{0}$ of $220 \mathrm{MeV}$, since it yields a cold neutron star mass-radius relationship in agreement with current observational and theoretical constraints (e.g., Demorest et al. 2010; Hebeler et al. 2010; Steiner et al. 2010).

We employ the Lattimer-Swesty EOS at densities above $10^{8} \mathrm{~g} \mathrm{~cm}^{-3}$, where $T \gtrsim 0.5 \mathrm{MeV}$ at all times in the core-collapse context and nuclear statistical equilibrium (NSE) holds. At lower densities, we employ the Timmes EOS (Timmes \& Arnett 1999) and assume that the matter is an ideal gas composed of electrons, positrons, photons, neutrons, protons, alpha particles, and heavy nuclei with the average $A$ and $Z$ given by the Lattimer-Swesty EOS at the transition density. This is an approximation and may lead to slightly incorrect pressures in non-NSE regions that result in changes in the collapse times for the silicon and carbon/oxygen shells. Ideally, a fully consistent treatment with multiple advected chemical species, a nuclear reaction network and transition in and out of NSE with an NSE network as proposed by Buras et al. (2006b) should be implemented. This, however, is beyond the scope of the present study.

Details on the EOS table and on the implementation of the contribution of electrons, positrons, and photons, as well as other details of the construction of the table are described in O'Connor \& Ott (2010). The table itself as well as table generation and interpolation routines are available at http://www.stellarcollapse.org.

\subsection{Neutrino Treatment}

We employ the approximate neutrino treatment of the open-source code GR1D (O'Connor \& Ott 2010), which was adapted to $3 \mathrm{D}$ and implemented in the module ZelmaniLeak by Ott et al. (2012). The source code is available from http://www.stellarcollapse.org.

Before core bounce, the primary neutrino emission process is electron capture on free and bound protons, leading to a reduction of the electron fraction $Y_{e}$ in the collapsing core. We include this effect and associated changes of the specific entropy in the approximate way proposed by Liebendörfer (2005). He showed, on the basis of 1D Boltzmann neutrino radiationhydrodynamics simulations, that $Y_{e}$ in the collapse phase can be well parameterized as a function of rest-mass density $\rho$. This parameterization shows only small variations with progenitor star and nuclear EOS. We employ an analytic $Y_{e}(\rho)$ fit to the results of $1 \mathrm{D}$ radiation-hydrodynamics collapse simulations of a $20 M_{\odot}$ solar-metallicity progenitor star of Woosley et al. (2002) obtained with the code and microphysics of Buras et al. (2006b). The same $Y_{e}(\rho)$ profile was used in Ott et al. (2007a, 2007b) and Ott et al. (2012).

In the late collapse phase, when neutrinos begin to be trapped in the inner core, and throughout the postbounce phase, momentum exchange between neutrinos and matter becomes non-negligible. The effect of this "neutrino stress" is naturally captured by the coupling of radiation and matter in full neutrino transport calculations (see, e.g., Müller et al. 2010). In our approximate treatment, we must include it explicitly. We assume that neutrino stress is relevant only above a fiducial trapping density of $2 \times 10^{12} \mathrm{~g} \mathrm{~cm}^{-3}$ and approximate the stress as the gradient of the neutrino Fermi pressure. The stress is then included as a source term in the GR hydrodynamics equations at each time-integration substep and the neutrino Fermi pressure is included in the stress-energy tensor (see Ott et al. 2007b and O'Connor \& Ott 2010 for details).

After core bounce, which we define as the time at which the specific entropy at the edge of the inner core reaches $3 k_{\mathrm{B}}$ baryon $^{-1}$, signaling shock formation, the simple $Y_{e}(\rho)$ approximation breaks down and fails to even qualitatively capture the effects of neutrino processes occurring in the postbounce phase. Dissociation of iron-group nuclei by the shock provides a sea of free protons for electrons to capture on, leading to the neutronization burst of electron neutrinos $\left(v_{e}\right)$ and a steep drop of $Y_{e}$ in the region just outside the nascent protoneutron star. High temperatures and low $Y_{e}$ in the lower postshock region allow for the appearance of positrons that capture on neutrons, leading to the emission of electron antineutrinos $\left(\bar{v}_{e}\right)$. High temperatures in the protoneutron star core lead to neutral-current pair emission of neutrinos of all species.

In order to capture the aforementioned processes and their effects in terms of cooling, heating, and deleptonization in the region behind the shock, we switch to the neutrino leakage scheme of O'Connor \& Ott (2010; based on the work of Rosswog et al. 2003 and Ruffert et al. 1996) at bounce. We consider three neutrino species, $v_{e}, \bar{v}_{e}$, and $v_{x}=\left\{v_{\mu}, \bar{v}_{\mu}, v_{\tau}, \bar{v}_{\tau}\right\}$, where we lump the heavy-lepton neutrinos together, since they participate only in neutral current processes and have very similar cross sections in the core-collapse supernova environment.

The leakage scheme provides approximate energy and number emission and absorption rates based on local thermodynamics and the optical depth in the postshock region. Neutrino absorption and emission are ignored outside the shock. The optical depth requires a non-local calculation, which we solve in a ray-by-ray way, computing an optical depth integral $\tau_{\nu_{i}}$ along radial rays cast into $\theta$ and $\varphi$ directions (see Figure 1 of Ott et al. 2012) from the origin. We then interpolate trilinearly in $(r, \theta, \varphi)$ to obtain the optical depth at the centers of Cartesian grid cells. Ideally, an optical depth calculation should be carried out into all directions from any given cell and the minimum value should be used as the optical depth of that cell (see, e.g., Ruffert et al. 1996). However, for situations that are spherical at zeroth order, like the one considered here, the computationally much cheaper ray-by-ray approach should be sufficient. In our simulations, we employ 37 rays in $\theta$, covering $[0, \pi]$, and 75 rays in $\varphi$, covering $[0,2 \pi]$. Each ray has 800 equidistant points to $\sim 600 \mathrm{~km}$ and 200 logarithmically spaced points covering $\sim 600-3000 \mathrm{~km}$.

We calculate local free neutrino energy $\left(Q_{v_{i}}^{\text {loc }}\right)$ and number $\left(R_{v_{i}}^{\text {loc }}\right)$ emission rates for the capture processes $p+e^{-} \rightarrow$ $v_{e}+n$ and $e^{+}+n \rightarrow \bar{v}_{e}+p$ and the thermal processes $e^{-} e^{+}$pair annihilation, plasmon decay, and nucleon-nucleon bremsstrahlung. Using the estimate of the optical depth $\tau_{v_{i}}$, we compute diffusive emission rates $Q_{v_{i}}^{\text {diff }}$ and $R_{v_{i}}^{\text {diff }}$ and obtain the final energy and number loss predicted by the leakage scheme by interpolating between free emission and diffusive emission rates,

$$
\chi_{\mathrm{eff}, v_{i}}^{\text {leak }}=\chi_{\mathrm{loc}, v_{i}}^{\text {leak }} /\left(1+\chi_{\mathrm{loc}, v_{i}}^{\text {leak }} / \chi_{\mathrm{diff}, v_{i}}^{\text {leak }}\right),
$$

where $\chi=Q$ for energy loss and $\chi=R$ for number loss (see Rosswog \& Liebendörfer 2003 and O'Connor \& Ott 2010 for definitions and details).

We approximately include neutrino heating by chargedcurrent absorption of $v_{e}$ and $\bar{v}_{e}$ on neutrons and protons, 
respectively. For this, we make use of a local heating function based on the derivations by Janka (2001),

$$
Q_{v_{i}}^{\text {heat }}=f_{\text {heat }} \frac{L_{v_{i}}(r)}{4 \pi r^{2}} S_{\nu}\left\langle\epsilon_{v_{i}}^{2}\right\rangle \frac{\rho}{m_{n}} X_{i}\left\langle\frac{1}{F_{v_{i}}}\right\rangle e^{-2 \tau_{v_{i}}} .
$$

Here $L_{v_{i}}$ is the neutrino luminosity incident from below, $S_{v}=0.25\left(1+3 \alpha^{2}\right) \sigma_{0}\left(m_{e} c^{2}\right)^{-2}$, where $\sigma_{0}$ is the fiducial weak interaction cross-section $\sim 1.76 \times 10^{-44} \mathrm{~cm}^{2}, \alpha=1.23$, and $m_{e} c^{2}$ is the electron rest mass energy in MeV. $\rho$ is the rest-mass density, $m_{n}$ is the neutron mass in grams, and $X_{i}$ is the neutron (or proton) mass fraction. $\left\langle\epsilon_{v_{i}}^{2}\right\rangle$ is the mean-squared energy of $v_{i}$ neutrinos. We approximate it by taking the matter temperature $T_{\mathrm{NS}, v_{i}}$ at the $v_{i}$ neutrinosphere (where $\tau_{v_{i}}=2 / 3$ ) and evaluating

$$
\left\langle\epsilon_{v_{i}}^{2}\right\rangle=T_{\mathrm{NS}, v_{i}}^{2} \frac{\mathcal{F}_{5}\left(\eta_{v_{i}, \mathrm{NS}}\right)}{\mathcal{F}_{3}\left(\eta_{v_{i}, \mathrm{NS}}\right)},
$$

where the $\mathcal{F}_{j}$ are Fermi integrals $\mathcal{F}_{j}(\eta)=\int_{0}^{\infty} d x x^{j}\left(e^{x-\eta}+1\right)^{-1}$, and $\eta_{v_{i}, N S}=\mu_{v_{i}, \mathrm{NS}}\left(k_{\mathrm{B}} T_{\mathrm{NS}, v_{i}}\right)^{-1}$, where $\mu_{v_{i}, \mathrm{NS}}$ is the chemical potential of neutrino species $v_{i}$ at its neutrino sphere. The factor $\left\langle F_{v_{i}}^{-1}\right\rangle$ is the mean inverse flux factor, which depends on details of the neutrino radiation field. We parameterize it as a function of optical depth $\tau_{\nu_{i}}$ based on the angle-dependent radiation fields of the neutrino transport calculations of Ott et al. (2008) and set $\left\langle F_{v_{i}}^{-1}\right\rangle=4.275 \tau_{v_{i}}+1.15$. While the true mean inverse flux factor will asymptote to 1 at infinity, this simple fit leads to values in the postshock region (we include heating only there) in agreement with Ott et al. (2008). Finally, the factor $e^{-2 \tau_{v_{i}}}$ is applied to strongly suppress heating at optical depth above unity. The leakage scheme implementation in Zelmani varies slightly from the original implementation in O'Connor \& Ott (2010, 2011). In O'Connor \& Ott (2010, 2011), leakage was calculated only inside the shock to avoid unnecessary calculations outside of the shock where little cooling or heating occurred. To facilitate easy implementation in Zelmani, where the angle-dependent shock radius is evaluated only infrequently, we have removed the explicit dependence on the shock radius and have replaced it with a condition on the mass fraction of heavy nuclei: we only calculate the heating and cooling terms where the heavy nuclei mass fraction is smaller than 0.5 or the density is higher than $10^{13} \mathrm{~g} \mathrm{~cm}^{-3}$.

We obtain the neutrinosphere locations and the thermodynamic conditions for Equation (3) from the rays used for the optical depth calculations. We also solve full leakage problems including heating along the rays to obtain an estimate for the incident luminosity $L_{v_{i}}$ needed by Equation (2). The estimates for $L_{v_{i}}$ and $\left\langle\epsilon_{v_{i}}^{2}\right\rangle$ are then interpolated between rays for the local leakage calculations in Cartesian grid cells.

All of the above leakage calculations are carried out operatorsplit after the fully coupled spacetime/hydro update and are first order in time. We find this to be sufficiently accurate and stable, due to the small time step imposed by the light travel time through the smallest cell. The energy and lepton number updates are applied to the fluid rest-frame quantities and we ignore velocity dependence or other relativistic effects in consideration of the overall very approximate nature of the leakage scheme. The computationally most expensive aspect of the leakage scheme is the interpolation of density, temperature, and electron fraction onto the rays. This interpolation is executed at every time step in the highly dynamic early postbounce phase. We later switch to carrying out this interpolation only every 16 fine grid time steps (corresponding to every $\sim 8 \times 10^{-6}$ s) while continuing to evaluate the local expressions at every time step.

\subsection{Initial Model}

We simulate core collapse and postbounce evolution in the nonrotating single-star $27 M_{\odot}$ solar-metallicity model $s 27$ of Woosley et al. (2002). We choose this particular model to facilitate comparisons with the recent 2D results of Müller et al. (2012a). As pointed out by Müller et al. (2012a), this progenitor has an iron-core mass ${ }^{14}$ of $\sim 1.5 M_{\odot}$ and a siliconshell mass of $\sim 0.18 M_{\odot}$. According to O'Connor \& Ott (2011), this progenitor, having a bounce compactness parameter $\left.\xi_{2.5}=2.5\left(R\left[M=2.5 M_{\odot}\right)\right] / 1000 \mathrm{~km}\right)^{-1}=0.233$, is a likely candidate for explosion via the neutrino mechanism. The recent work of Ugliano et al. (2012) predicts a higher failed core-collapse supernova rate for the solar metallicity model set of Woosley et al. (2002) than the work of O'Connor \& Ott (2011). However, they also predict that this particular presupernova model is a progenitor of a successful neutrinodriven core-collapse supernova.

Using the spherically-symmetric GR1D code of O'Connor $\&$ Ott $(2010,2011)$ and modifying its leakage scheme to be identical to what we use in Zelmani, we find that $f_{\text {heat }}=1.18$ is the critical value of the scaling factor in Equation (2) to drive an explosion that sets in at late times after multiple cycles of radial shock oscillations. $f_{\text {heat }}=1.28$ is required to drive an explosion without shock oscillations that sets in at $\sim 150 \mathrm{~ms}$ after bounce.

We map model $s 27$ to our 3D grid under the assumption that the $1 \mathrm{D}$ profile data represent cell averages and use the radii of cell centers for interpolation. The initial spacetime is set up under the assumption of spherical symmetry and weak gravity, using the Newtonian line element without distinction between areal and isotropic radius.

\section{RESULTS}

\subsection{Overall Postbounce Evolution}

We simulate core collapse and bounce of the $27 M_{\odot}$ progenitor in full 3D with AMR, adding refinement levels as the collapse toward a protoneutron star proceeds (see Section 2.2). Core bounce, defined as the time when the entropy at the edge of the inner core reaches $3 k_{\mathrm{B}}$ baryon $^{-1}$, occurs at $\sim 299 \mathrm{~ms}$. At bounce, we switch from the $Y_{e}(\rho)$ parameterization of Liebendörfer (2005) to the leakage/heating scheme described in Section 2.4. This scheme includes a scaling factor $f_{\text {heat }}$ in the neutrino energy deposition rate (Equation (2)). We carry out four long-term postbounce simulations, choosing $f_{\text {heat }}=\{1.00,1.05,1.10,1.15\}$ to study the influence of changes in the heating rate on the postbounce evolution. All models are labeled according to their value of $f_{\text {heat }}$. For example, $s 27 f_{\text {heat }} 1.00$ is the model with $f_{\text {heat }}=1.00$. All models are evolved to $\gtrsim 150 \mathrm{~ms}$ after bounce and for as long as our computer time allocations allow at a cost of $\sim 25,000$ CPU hours per millisecond of physical postbounce time (see Table 1).

In the top panel of Figure 2, we show the angle-averaged shock radius as a function of time in the four simulated models. After the early dynamic expansion phase, shock expansion stagnates and the shock stalls at $100-130 \mathrm{~km}$ about $40 \mathrm{~ms}$ after bounce. Up to this point, the evolution is virtually independent of $f_{\text {heat }}$. In the subsequent period of quasi-stationary evolution, the gain layer develops and neutrino heating drives a secular shock expansion.

\footnotetext{
14 We define the iron-core mass as the mass coordinate that has a $Y_{e}$ of 0.495 .
} 


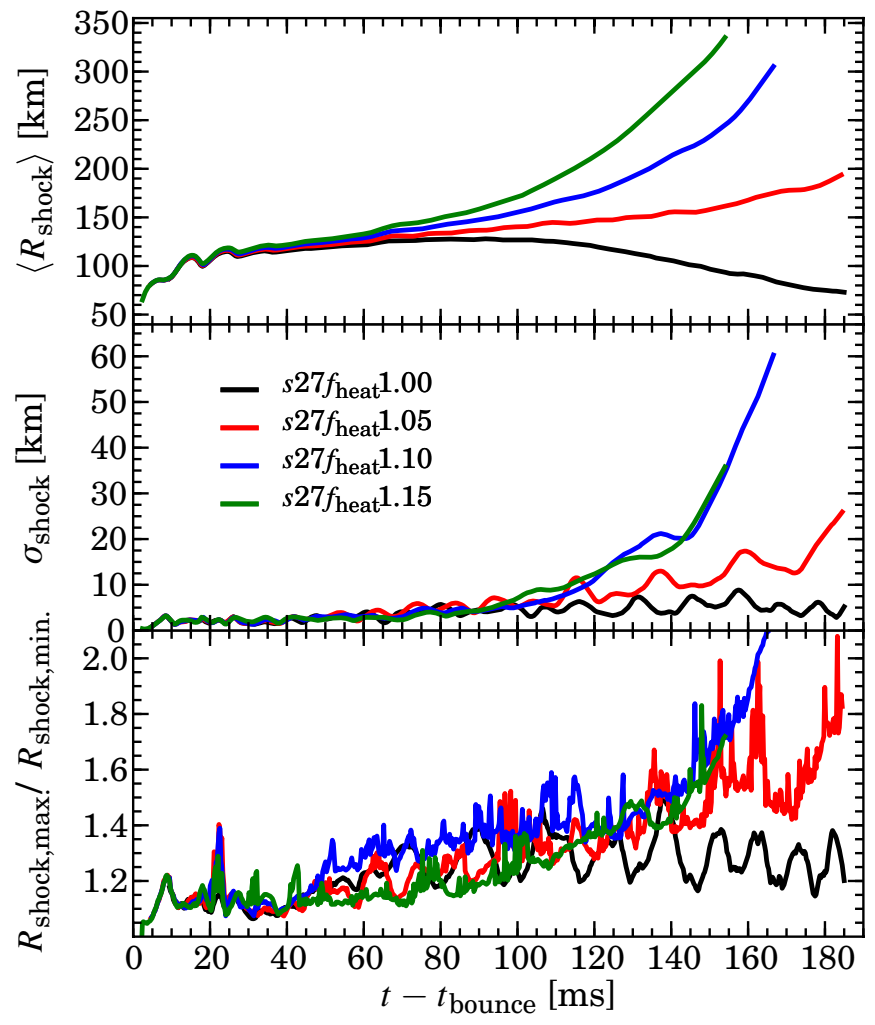

Figure 2. Global evolution of the shock in all models. Top panel: average shock radii $\left\langle R_{\text {shock }}\right\rangle$. Center panel: standard deviation $\sigma_{\text {shock }}=\left[(4 \pi)^{-1} \int d \Omega\left[R_{\text {shock }}-\right.\right.$ $\left.\left.\left\langle R_{\text {shock }}\right\rangle\right]^{2}\right]^{1 / 2}$ of the shock radii. Bottom panel: ratio of maximum to minimum shock radius. The shock radii of models with $f_{\text {heta }} \geqslant 1.05$ exhibit positive trends in their average shock radii and have growing $\sigma_{\text {shock }}$ and ratios between maximum and minimum shock radius. Model $s 27 f_{\text {heat }} 1.00$ 's shock radius starts decreasing at $\sim 100 \mathrm{~ms}$ after bounce and its $\sigma_{\text {shock }}$ and $\min /$ max shock radii ratios oscillate around moderate values.

(A color version of this figure is available in the online journal.)

Table 1

Key Simulation Parameters and Results

\begin{tabular}{lccccccc}
\hline \hline Model & $\begin{array}{c}f_{\text {heat }} d x_{\text {shock }} \\
(\mathrm{km})\end{array}$ & $\begin{array}{c}d \theta, d \phi \\
\text { at } 100 \mathrm{~km}(\mathrm{~ms}) \\
(\mathrm{deg})\end{array}$ & $\begin{array}{c}t_{\text {end }} \\
R_{\text {shock,max }} \\
\text { at } t_{\text {end }} \\
(\mathrm{km})\end{array}$ & $\begin{array}{r}R_{\text {shock,av }} \\
\text { at } t_{\text {end }} \\
(\mathrm{km})\end{array}$ & $\begin{array}{r}R_{\text {shock,min }} \\
\text { at } t_{\text {end }} \\
(\mathrm{km})\end{array}$ \\
\hline$s 27 f_{\text {heat }} 1.00$ & 1.00 & 1.48 & 0.85 & 184 & 82 & 71 & 62 \\
$s 27 f_{\text {heat }} 1.05$ & 1.05 & 1.48 & 0.85 & 192 & 259 & 189 & 152 \\
$s 27 f_{\text {heat }} 1.10$ & 1.10 & 1.48 & 0.85 & 165 & 428 & 306 & 204 \\
$s 27 f_{\text {heat }} 1.15$ & 1.15 & 1.48 & 0.85 & 154 & 432 & 336 & 267 \\
\hline
\end{tabular}

Notes. $f_{\text {heat }}$ is the scaling factor in the neutrino heating rate (Equation (2)), $d x_{\text {shock }}$ is the minimum linear resolution covering the shock and the region interior to it, $d \theta, d \phi$ at $100 \mathrm{~km}$ is the effective angular resolution at a radius of $100 \mathrm{~km}, t_{\text {end }}$ is the time after core bounce at which the simulation is stopped, and $R_{\text {shock,max }}, R_{\text {shock,av }}$, and $R_{\text {shock,min }}$ are the final maximum, average, and minimum shock radius, respectively.

The neutrino luminosity emitted from the protoneutron star core and provided by accretion is identical in all models. Hence, as shown in the top panels of Figure 3, there is a monotonic increase with $f_{\text {heat }}$ in the net neutrino heating rate $Q_{\text {net }}$ and in the heating efficiency $\eta=Q_{\text {net }}\left(L_{v_{e}}+L_{\bar{v}_{e}}\right)^{-1}$, where we use the angle-averaged luminosities at the base of the gain layer. Varying $f_{\text {heat }}$ by a moderate $15 \%$ from 1.00 to 1.15 results in $\sim 100 \%$ more total net heating, since the increase in the local energy deposition rate results in an expanded gain layer with

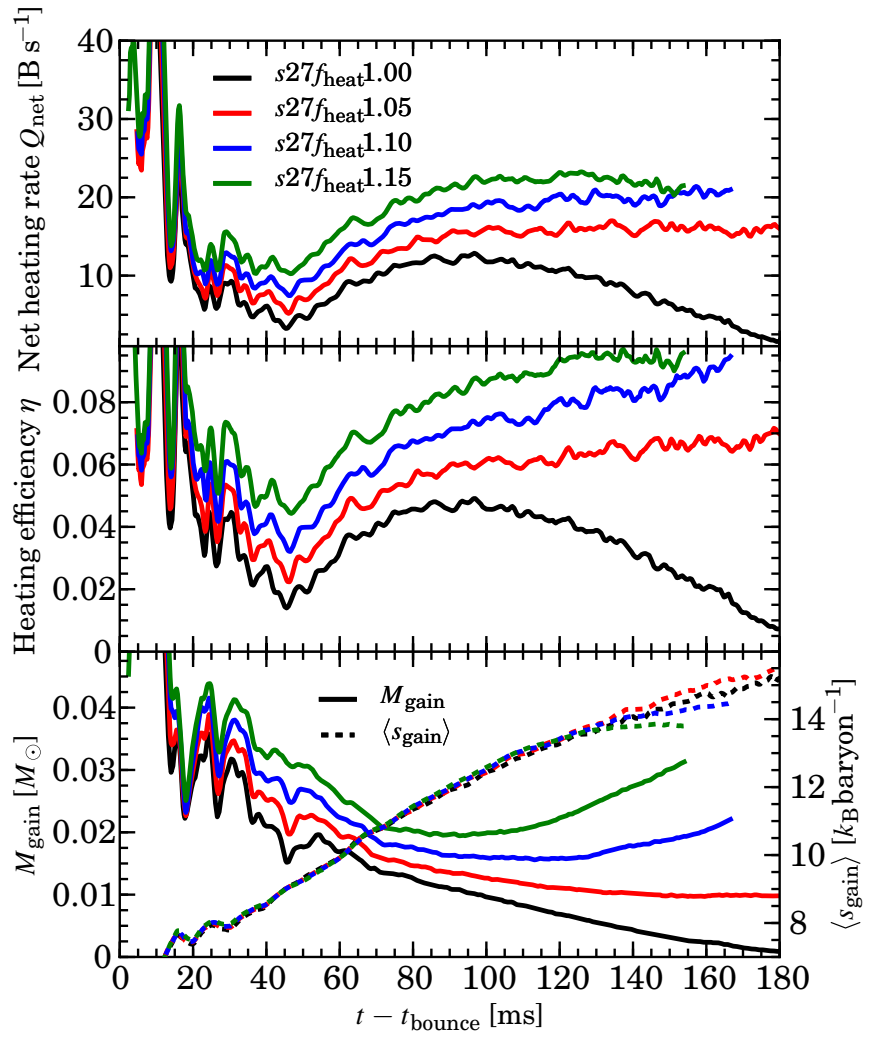

Figure 3. Evolution of key integral quantities indicative for the strength of neutrino heating. Top panel: net neutrino heating rate $Q^{\text {net }}$ (total heating minus total cooling). Center panel: heating efficiency $\eta$ defined as the net heating rate divided by the sum of the $v_{e}$ and $\bar{v}_{e}$ angle-averaged luminosities incident below the gain layer. Bottom panel: mass in the gain layer (left ordinate) and densityweighted average specific entropy in the gain layer (right ordinate). Heating rate, efficiency, and mass in the gain layer all increase monotonically with increasing heating scaling factor $f_{\text {heat }}$. Interestingly, the specific entropy average $\left\langle s_{\text {gain }}\right\rangle$ in the gain layer does not exhibit such a dependence on $f_{\text {heat }}$ and the $\left\langle s_{\text {gain }}\right\rangle$ curves of all models are nearly identical until $\gtrsim 100 \mathrm{~ms}$ after bounce, at which point the overall hydrodynamic evolutions have diverged.

(A color version of this figure is available in the online journal.)

more mass that is able to absorb net neutrino energy (cf. bottom panel of Figure 3).

The quantitative differences in neutrino energy deposition translate to qualitative differences in the shock evolution. In model $s 27 f_{\text {heat }} 1.00$, which has the least heating, shock stagnation turns into recession and the average shock radius decreases to $\sim 70 \mathrm{~km}$ at the end of the simulation. The situation is very different in models $s 27 f_{\text {heat }} 1.10$ and $s 27 f_{\text {heat }} 1.15$, which both show expanding average shock radii, surpassing $300 \mathrm{~km}$ at the end of their simulations and trending toward explosion. Model $s 27 f_{\text {heat }} 1.05$ is somewhere in between, but has a slowly, but steadily increasing average shock radius that reaches $\sim 190 \mathrm{~km}$ at the end of the simulation.

The center and bottom panels of Figure 2 display simple measures of the asphericity of the shock: $\sigma_{\text {shock }}$, the angular standard deviation of the shock radius, and $R_{\text {shock,max }} / R_{\text {shock,min }}$, the ratio of maximum to minimum shock radius. Both quantities show an initial local maximum at $\sim 8 \mathrm{~ms}$ after bounce, which is due to an initial transient large $\ell=4$ deformation of the shock front caused by the Cartesian grid employed in our simulations. We will discuss this further in Section 3.3. In the first $40 \mathrm{~ms}$ after bounce, all models show very similar small deviations of the shock from spherical symmetry. Differences between models begin to be apparent at the same time their average shock radii 


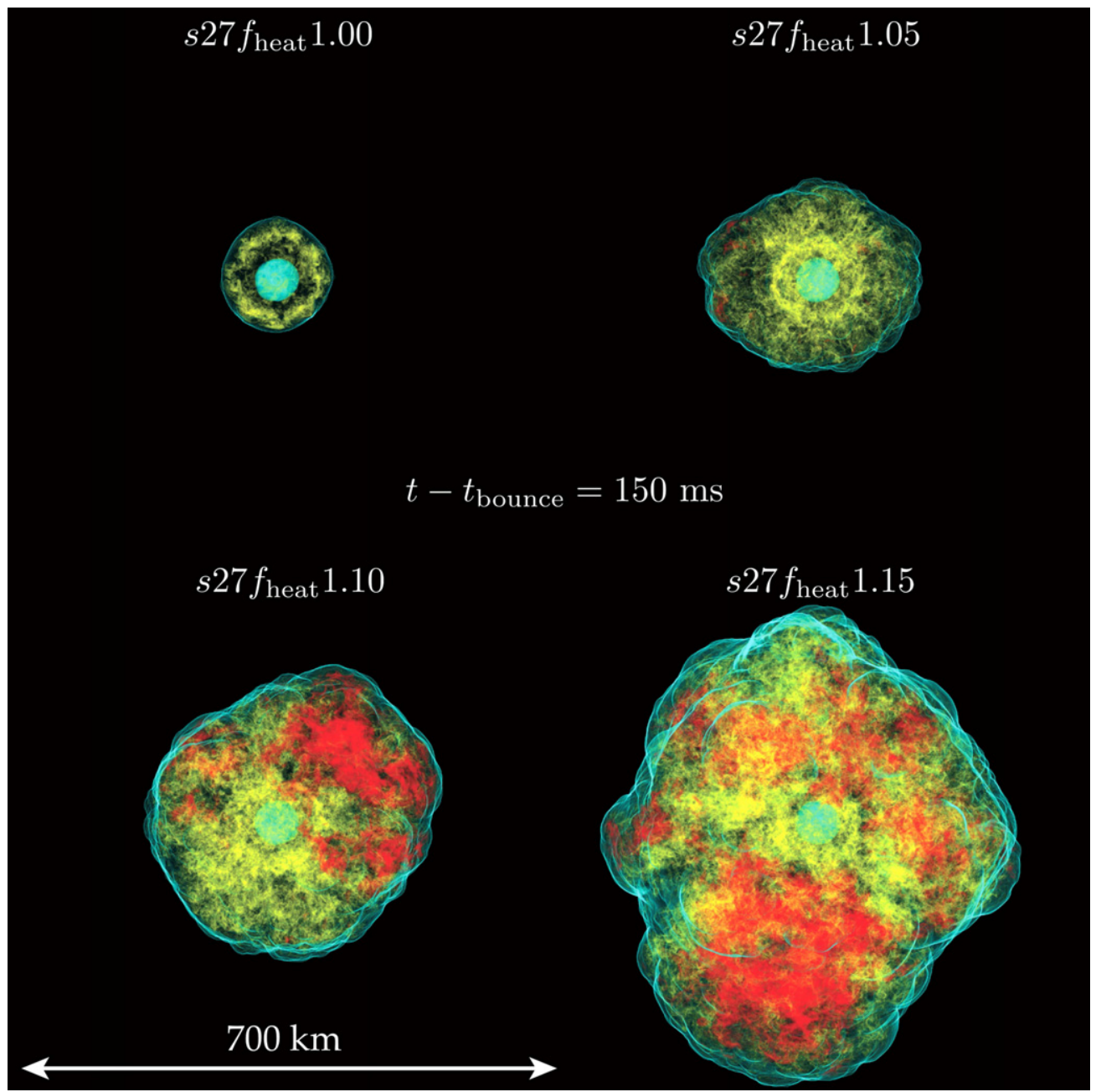

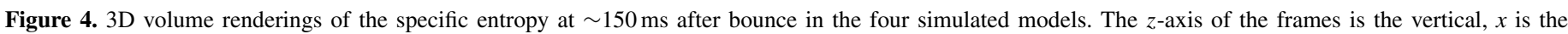

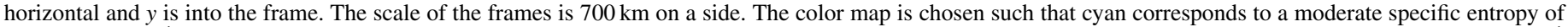

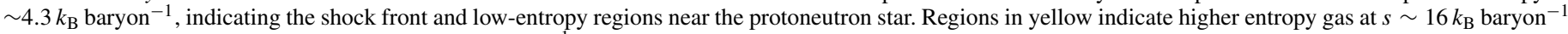

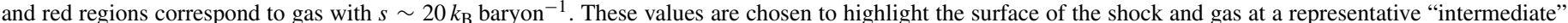

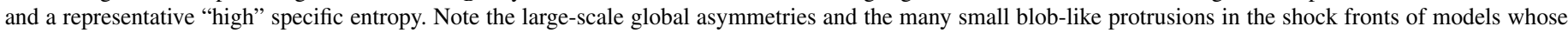
shock has reached large radii.

(A color version of this figure is available in the online journal.)

begin to diverge. Models $s 27 f_{\text {heat }} 1.10$ and $s 27 f_{\text {heat }} 1.15$ exhibit very large asymmetries with $\sigma_{\text {shock }} \sim 30 \mathrm{~km}$ and almost a factor of two in radius between maximum and minimum shock radius at the end of their simulations (see Table 1 for final minimum, maximum, and average shock radii for all models). Model $s 27 f_{\text {heat }} 1.05$ also shows growing asymmetry with increasing postbounce time, similar to the models with $f_{\text {heat }}=1.10$ and 1.15 , but, at least in $\sigma_{\text {shock }}$, a periodicity is visible, which is lacking completely or is occurring at a much smaller level in the two models with larger $f_{\text {heat }}$. In model $s 27 f_{\text {heat }} 1.00$, which does not show a positive trend in its shock radius, the deviations of the shock from sphericity remain small and maximum and minimum shock radius differ, on average, by $\sim 20 \%$ and this average difference does not grow until the end of the simulation. There is, however, clear oscillatory behavior (with a short period of $\sim 15 \mathrm{~ms}$ ) in this model's shock radius variations, which may be indicative of SASI activity. We shall investigate this further in Section 3.3.

In Figure 4, we present volume renderings of the specific entropy at $\sim 150 \mathrm{~ms}$ after bounce for all four models. The renderings are all plotted at the same scale to emphasize the differences in shock radius and 3D geometry between the models. The color map and rendering opacity are chosen to emphasize (1) regions with specific entropy of $\sim 4.3 k_{\mathrm{B}}$ baryon $^{-1}$ (cyan), (2) regions with a representative "intermediate" specific entropy of $\sim 16 k_{\mathrm{B}}$ baryon $^{-1}$ (yellow), and, (3) regions with a representative "high" specific entropy of $\sim 20 k_{\mathrm{B}}$ baryon $^{-1}$ (red). Red and yellow thus mark gas in the high-entropy gain layer, while cyan indicates the shock front and an iso-entropy surface at the edge of the protoneutron star. While the shock appears nearly spherical in model $s 27 f_{\text {heat }} 1.00$, it is clearly deformed in model $s 27 f_{\text {heat }} 1.05$, and strongly so in models $s 27 f_{\text {heat }} 1.10$ and $s 27 f_{\text {heat }} 1.15$. One also notes that in the latter two models the highest-entropy gas is concentrated in the region of greatest expansion while it is more evenly spread out in the other models. The shock deformation in these models is clearly dominated by low- $\ell$ modes, but there is still much smaller-scale structure in the form of protrusions caused by rising hot gas bubbles that push out the shock front at local scales. The overall morphology of the expanding shock fronts seen in these models is similar 

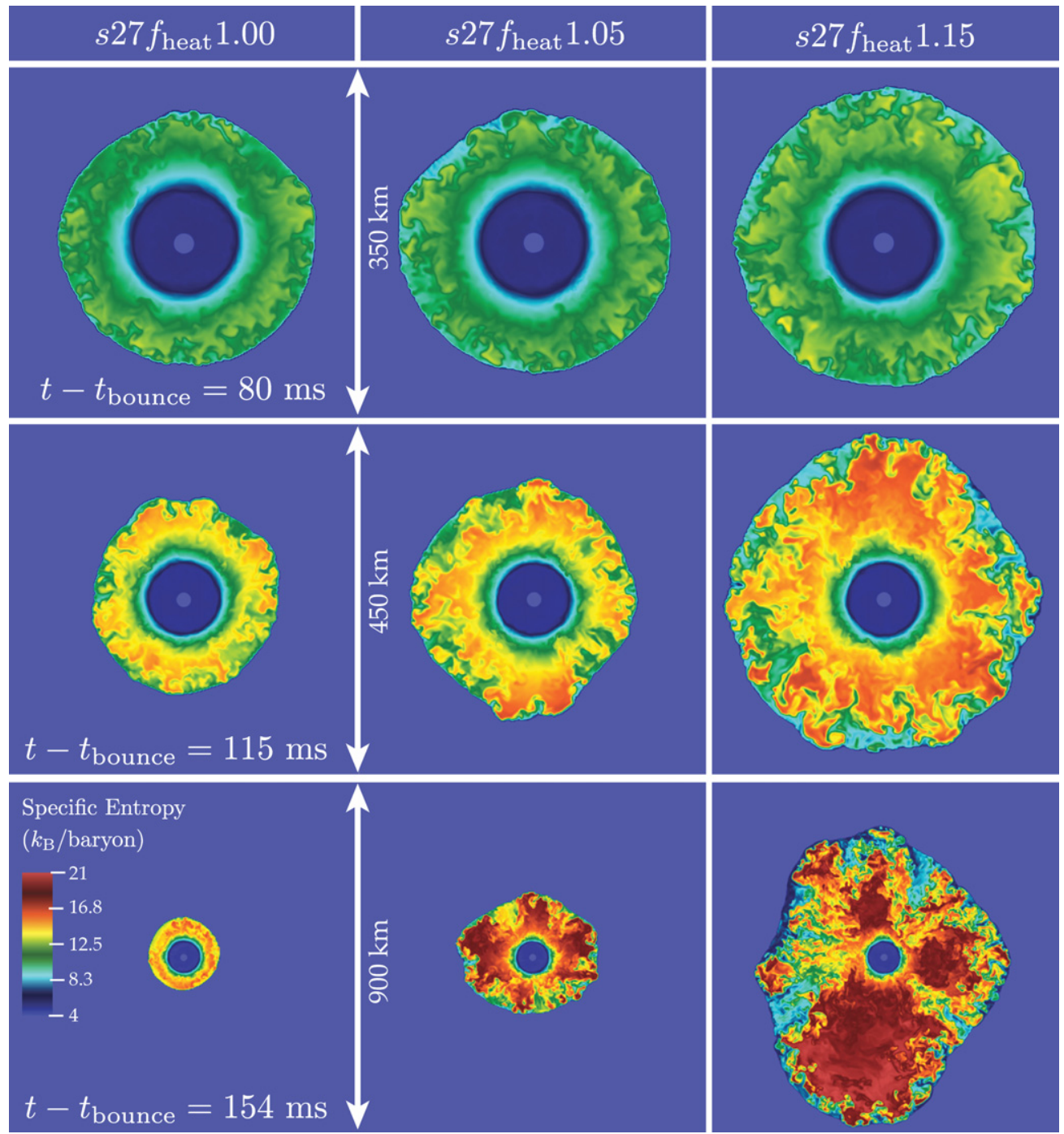

Figure 5. Color maps of the specific entropy in the $x-z$ plane in models $s 27 f_{\text {heat }} 1.00$ (left column), $s 27 f_{\text {heat }} 1.05$ (center column), and $s 27 f_{\text {heat }} 1.15$ (right column) at 80,115 , and $154 \mathrm{~ms}$ after core bounce. The linear scales of the three vertical panels are 350, 450, and $900 \mathrm{~km}$ at these three times. The values of the specific entropy in the convectively unstable gain region increase with time in all simulations. Model $s 27 f_{\text {heat }} 1.00$ exhibits a stagnant shock and only small deviations from sphericity. The average shock radius is secularly growing in model $s 27 f_{\text {heat }} 1.05$ with slightly stronger neutrino heating and the shock is more aspherical. Model $s 27 f_{\text {heat }} 1.15$ is on track to explosion and exhibits, at $154 \mathrm{~ms}$ after bounce, a strongly deformed shock with a single large high-entropy bubble.

(A color version of this figure is available in the online journal.)

to what was found by Dolence et al. (2013) in exploding 3D Newtonian light-bulb models of a $15 M_{\odot}$ progenitor, but their shock fronts appear to have less small-scale structure than ours (cf. their Figure 20).

Figure 5 depicts color maps of 2D $x-z$ slices of the specific entropy in models $s 27 f_{\text {heat }} 1.00, s 27 f_{\text {heat }} 1.05$, and $s 27 f_{\text {heat }} 1.15$ at 80,115 , and $154 \mathrm{~ms}$ after bounce. Model $s 27 f_{\text {heat }} 1.10$ is not shown, but is overall very similar to model $s 27 f_{\text {heat }} 1.15$. The evolution toward large shock radii, large-scale shock deformation, and peak specific entropies of $\gtrsim 20 k_{\mathrm{B}}$ is obvious in the slices belonging to models $s 27 f_{\text {heat }} 1.05$ and $s 27 f_{\text {heat }} 1.15$. In the latter, at $154 \mathrm{~ms}$, one notes a large high-entropy area subtending an angle of $\sim 30^{\circ}$ and ranging from the gain radius out to the shock, which has the overall greatest radii in this region. This is consistent with the volumetric view of this model at approximately the same time, shown in Figure 4.

In the bottom panel of Figure 3, we plot the density-weighted average of the specific entropy in the gain layer $\left\langle s_{\text {gain }}\right\rangle$ (dashed lines; right ordinate). While the heating rates differ strongly between the models, their $\left\langle s_{\text {gain }}\right\rangle$ remain very similar until $\sim 100 \mathrm{~ms}$ after bounce and $\left\langle s_{\text {gain }}\right\rangle \sim 12 k_{\mathrm{B}}$ baryon $^{-1}$. The top row of Figure 5 shows that the peak entropy reached in the gain layer is very comparable among the three displayed models at $80 \mathrm{~ms}$ after bounce. At $115 \mathrm{~ms}$ and, in particular, at $154 \mathrm{~ms}$, the situation is different. The models with increasing shock radii and shock deformations develop large regions with specific entropies in excess of $20 k_{\mathrm{B}}$ baryon $^{-1}$ and large spatial variations. In model $s 27 f_{\text {heat }} 1.15$, at $154 \mathrm{~ms}$ after bounce, the expanding deformed shock has already swept up cold gas that now moves through the gain layer, leading to a decreasing $\left\langle s_{\text {gain }}\right\rangle$ in this model. This is consistent with the decrease in $\left\langle s_{\text {gain }}\right\rangle$ seen at the onset of explosion in the 3D and 2D simulations of Hanke et al. (2012) and Dolence et al. (2013). At the same postbounce time, the shock in model $s 27 f_{\text {heat }} 1.00$ has receded to $\sim 90 \mathrm{~km}$ and the distribution of specific entropy behind it is much more uniform than in the other models. Its average entropy continues to increase despite the decrease in net heating (cf. top panel of Figure 3). This is due to the combined effect of smaller shock 
radii and small deformation of the shock front. The average specific entropy in model $s 27 f_{\text {heat }} 1.05$ also grows, since its net heating rate continues to stay high while its shock deformation is still moderate and shock expansion has not yet become dynamical.

\subsection{Protoneutron Star, Neutrino Emission, and Thermodynamics of the Postshock Region}

The three-species leakage/heating scheme employed in our simulations goes beyond the MB08 light-bulb approach taken by many recent 3D hydrodynamic studies (e.g., Nordhaus et al. 2010; Hanke et al. 2012; Burrows et al. 2012; Murphy et al. 2012; Dolence et al. 2013). These simulations use analytic cooling functions and neglect important protoneutron star cooling by $v_{x}$. They also do not take into account changes of the electron fraction $Y_{e}$ after bounce (Hanke et al. 2012) or do so only via a parameterization of $Y_{e}(\rho)$, which cannot account for the strong deleptonization in the region behind the shock due to electron capture on free protons. Neutrino heating is realized in these simulations by an analytic heating function with spatially and temporally constant neutrino temperature and luminosity. An important consequence of these approximations is that accreted material settling onto the protoneutron star cannot sufficiently cool, deleptonize and contract (Hanke et al. 2012; Müller et al. 2012a). This, in turn, results in too large shock radii and low advection speeds through the convectively unstable gain layer that may artificially favor the growth of convection over SASI (Scheck et al. 2008; Foglizzo et al. 2006; Müller et al. 2012a). Our leakage/heating scheme is designed specifically to overcome these limitations at little additional computational cost. We take into account cooling by $v_{e}, \bar{v}_{e}$, and $v_{x}$, account for the change in electron fraction by $v_{e}$ and $\bar{v}_{e}$ emission and absorption. Our heating prescription uses the true $v_{e}$ and $\bar{v}_{e}$ luminosities available at a given position for heating (as computed by leakage/heating at smaller radii) and the mean-squared neutrino energies entering the heating rate are determined by assuming blackbody emission from the $v_{e}$ and $\bar{v}_{e}$ neutrinospheres, taking the time-changing thermodynamic locations on these surfaces into account.

While clearly not as sophisticated as recent gray multidimensional (e.g., Scheck et al. 2008; Müller et al. 2012c; Kuroda et al. 2012) or energy-dependent (e.g., Ott et al. 2008; Marek \& Janka 2009; Müller et al. 2012a, 2012b; Takiwaki et al. 2012) neutrino radiation-hydrodynamics calculations, the goal of our approach is to capture the essential qualitative features correctly and reproduce quantitative results approximately. In the following, we investigate the extent to which our scheme lives up to its premise.

In Figure 6, we plot, for all four models, the time evolutions of the baryonic mass inside the $10^{11} \mathrm{~g} \mathrm{~cm}^{-3}$ density isosurface (top panel, left ordinate), the angle-averaged accretion rate measured outside the shock (top panel, right ordinate), the angle-averaged coordinate radius of the $10^{11} \mathrm{~g} \mathrm{~cm}^{-3}$ density isosurface (center panel), and the angle-averaged $v_{e}, \bar{v}_{e}$, and $v_{x}$ neutrinosphere radii (where $\tau_{v_{i}}=1$; bottom panel). The evolutions of protoneutron star mass and radius, and of the accretion rate are very similar in all models. The radius if the $10^{11} \mathrm{~g} \mathrm{~cm}^{-3}$ isosurface, which we define as the surface of the protoneutron star following Müller et al. (2012b), shrinks from $\sim 70 \mathrm{~km}$ early after bounce to $40 \mathrm{~km}$ at $180 \mathrm{~ms}$ after bounce. At the same time, the enclosed baryonic mass increases from $\sim 1.15 M_{\odot}$ to $1.55 M_{\odot}$. If accretion suddenly stopped completely at $180 \mathrm{~ms}$, the gravitational mass of the final, cold

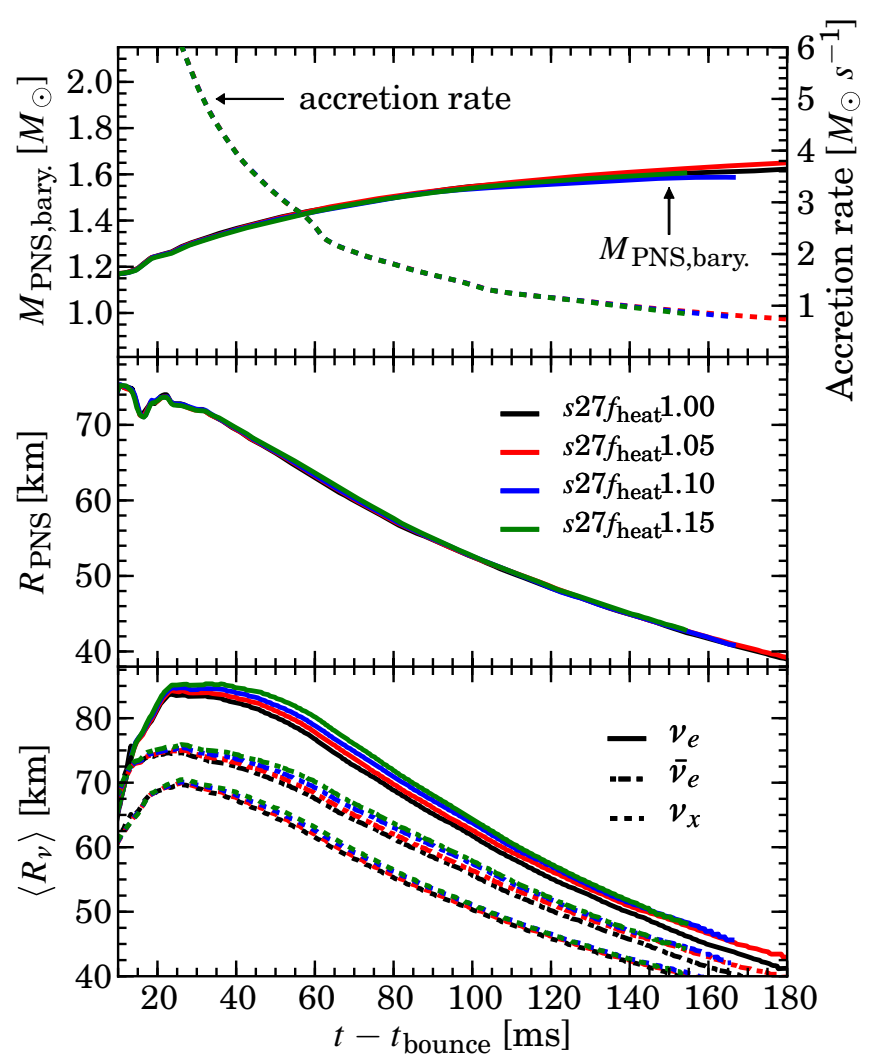

Figure 6. Top panel: evolution of the mass accretion rate measured at the shock (right ordinate) and baryonic mass of the protoneutron star enclosed by the $10^{11} \mathrm{~g} \mathrm{~cm}^{-3}$ density isosurface (left ordinate). Center panel: evolution of the protoneutron star radius, defined as the location of the $10^{11} \mathrm{~g} \mathrm{~cm}^{-3}$ point on the angle-averaged rest-mass density profile. The protoneutron star contracts as neutrino-cooling and deleptonizing material is settling on its surface. This is expected from 1D and 2D neutrino radiation-hydrodynamics simulations (cf. Müller et al. 2012b), but is not captured by the simple MB08 light-bulb approach (S. Richers et al. 2013, in preparation). Bottom panel: evolution of the gray, angle-averaged neutrinosphere radii $\left\langle R_{v}\right\rangle$ as predicted by the leakage scheme. The well-known hierarchy $R_{v_{e}}>R_{\bar{v}_{e}}>R_{v_{x}}$ is reproduced and the neutrinospheres follow the contraction of the protoneutron star as expected from full radiation-hydrodynamics simulations (e.g., Janka et al. 2007).

(A color version of this figure is available in the online journal.)

neutron star would be $\sim 1.4 M_{\odot}$ (Lattimer \& Prakash 2001). The increase in mass and decrease in radius of the protoneutron star seen in our simulations is qualitatively consistent with the findings of Müller et al. (2012b) and Buras et al. (2006a) for different progenitors. Müller et al. (2012a), who studied the $s 27$ progenitor, do not show these quantities. Hence, a direct quantitative comparison is not possible.

The angle-averaged neutrinosphere radii given in the lower panel of Figure 6 show that the leakage scheme correctly reproduces the well known hierarchy $R_{v_{e}}>R_{\bar{v}_{e}}>R_{\nu_{x}}$ of neutrinosphere radii in the postbounce pre-explosion phase (e.g., Janka et al. 2007). One notes that models with larger $f_{\text {heat }}$ have slightly larger neutrinosphere radii. We attribute this to their somewhat hotter postshock regions, resulting in higher opacity.

Model $s 27 f_{\text {heat }} 1.05$ is intermediate between model $s 27 f_{\text {heat }} 1.00$ that fails to explode in the simulated time and models $s 27 f_{\text {heat }} 1.10$ and $s 27 f_{\text {heat }} 1.15$, which have rapidly increasing shock radii at the end of their simulations. We choose $s 27 f_{\text {heat }} 1.05$ as our representative model and show, in Figure 7, angle-averaged profiles of its specific entropy, temperature, electron fraction, and rest-mass density at 40,80,120, and $140 \mathrm{~ms}$ after bounce. The smoothness of the profiles is due entirely to 


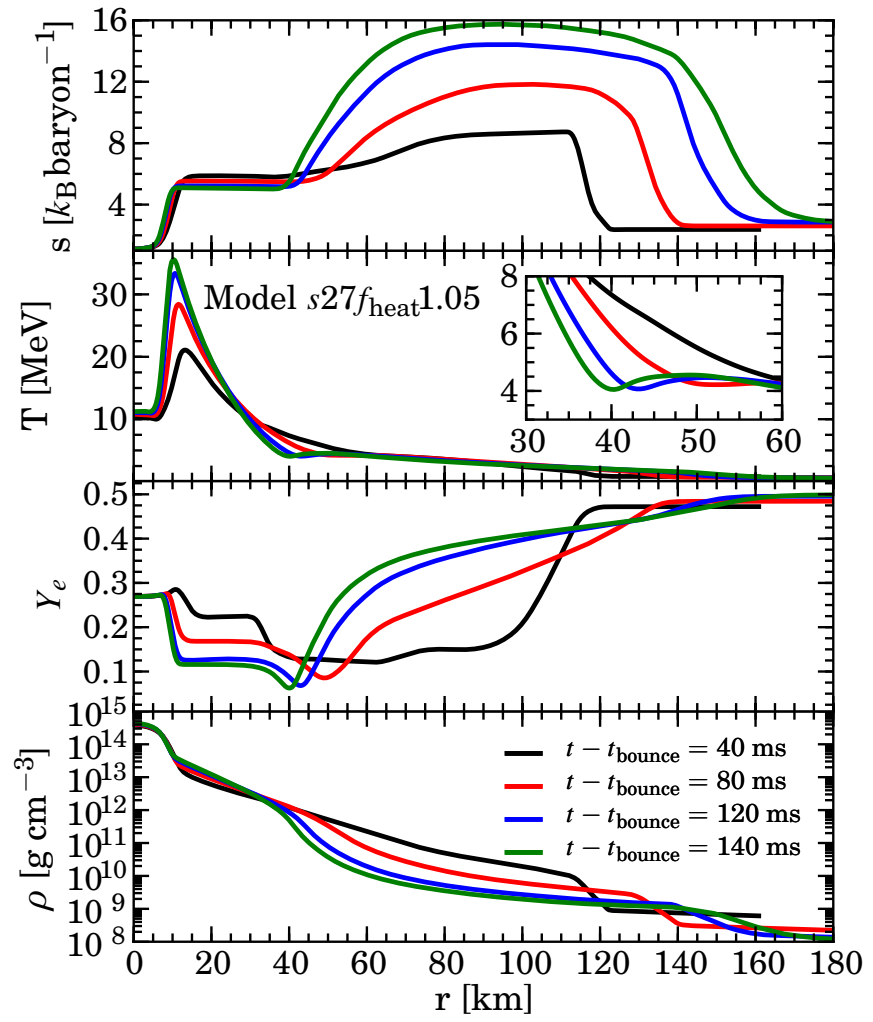

Figure 7. Angle-averaged profiles of specific entropy $s$ (top panel), temperature $T$ (second panel), electron fraction $Y_{e}$ (third panel) and the rest-mass density $\rho$ (bottom panel) at representative postbounce times in model $s 27 f_{\text {heat }} 1.05$. The data are taken from the AMR level encompassing the shock and, hence, do not extend to the full $180 \mathrm{~km}$ shown at early times. The smoothness of the curves is due entirely to the angle averaging. The profiles show the progressive deleptonization and contraction of the outer protoneutron star and the development of the high-entropy gain layer as is expected from full radiationhydrodynamics simulations (e.g., Buras et al. 200a, 2006b; Lentz et al. 2012; Müller et al. 2012b). Note, however, that our leakage/heating scheme tends to somewhat overestimate cooling and deleptonization at optical depths of a few, leading to a dip in $Y_{e}$ and a local temperature minimum around $40 \mathrm{~km}$. This temperature minimum is shown in a zoomed-in inset in the temperature panel.

(A color version of this figure is available in the online journal.)

angle averaging. The overall qualitative behavior of all quantities is as expected from more complex radiation-hydrodynamics simulations (cf. Buras et al. 2006b and Figure 5 of Dessart et al. 2006). The radial extent and specific entropy of the gain layer increase with time, while the changes in the specific entropy below $\sim 40 \mathrm{~km}$ simply reflect protoneutron star contraction. The latter is also well captured by the rising temperature at the protoneutron star edge, indicating compression. The strong deleptonization of the postshock region caused by the $v_{e}$ neutronization burst shortly after bounce is still visible in the $Y_{e}$ profile at $40 \mathrm{~ms}$ after bounce. The outer postshock region re-leptonizes over time due to a slight dominance of $v_{e}$ over $\bar{v}_{e}$ absorption in the gain layer. In the lower postshock region ( $R \sim 10-45 \mathrm{~km}$ ), neutrino cooling and deleptonization continue and, as expected from more accurate neutrino transport calculations, a strong negative lepton gradient develops that may drive protoneutron star convection (e.g., Dessart et al. 2006; Buras et al. 2006a).

The top panel of Figure 8 shows the total luminosities of $v_{e}, \bar{v}_{e}$, and $v_{x}$ as predicted by our leakage/heating scheme for models $s 27 f_{\text {heat }} 1.05$ and $s 27 f_{\text {heat }} 1.15$, which we take as representative examples. Differences between these models are minor and due to the greater heating in model $s 27 f_{\text {heat }} 1.15$. Since Müller et al.

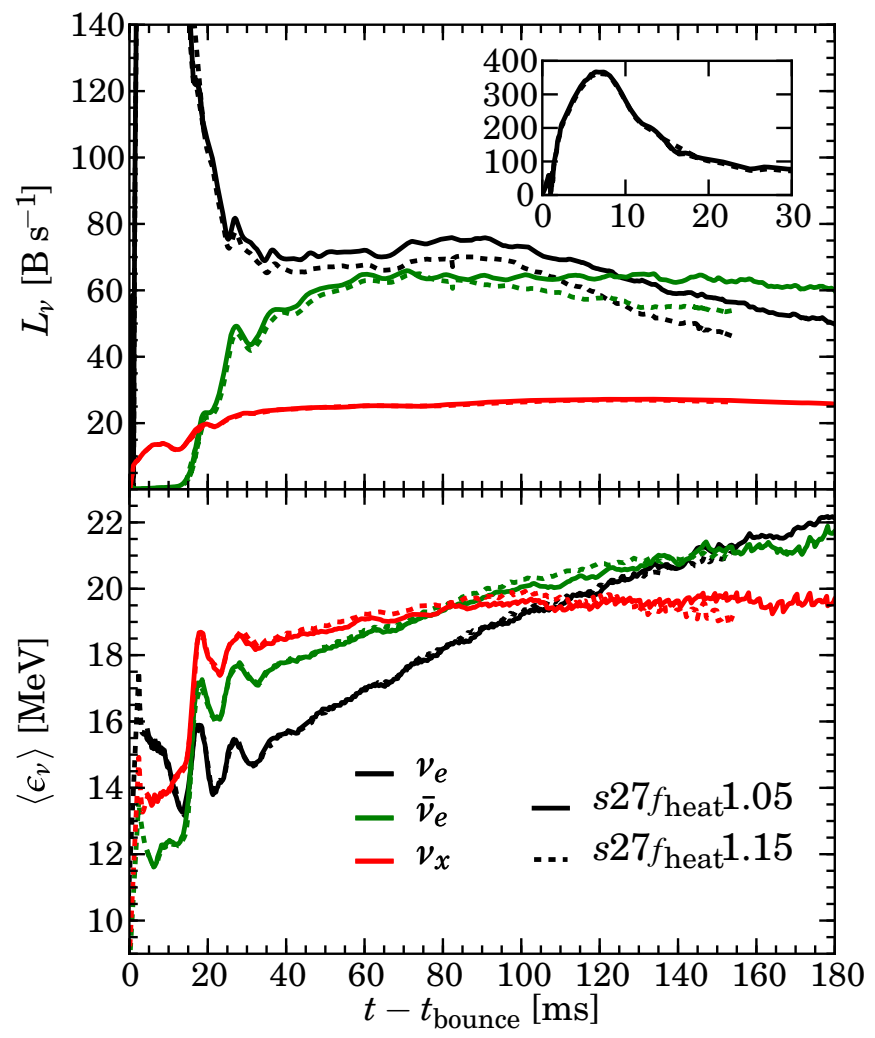

Figure 8. Top panel: $v_{e}, \bar{v}_{e}$, and $v_{x}$ luminosities as a function of postbounce time in models $s 27 f_{\text {heat }} 1.05$ (solid lines) and $s 27 f_{\text {heat }} 1.15$ (dashed lines) as representative examples of our model set. The $v_{e}$ and $\bar{v}_{e}$ luminosities in model $s 27 f_{\text {heat }} 1.15$ are somewhat smaller due to the strong charged-current absorption in this model. The inset plot shows the $v_{e}$ deleptonization peak. Comparing the $L_{v_{i}}$ shown here with those provided for the same progenitor in Figure 8 of Müller et al. (2012a) demonstrates that our much more approximate neutrino treatment still yields luminosities that agree within $\sim 20 \%$ with the results of true radiation-hydrodynamics simulations. Bottom panel: evolution of the mean neutrino energies $\left\langle\epsilon_{v_{i}}\right\rangle$ in the same models, obtained via the assumption of blackbody emission at the respective neutrinospheres. After the transient very early postbounce phase, the usual hierarchy of mean neutrino energies is established. At $\gtrsim 80-100$, ms after bounce, the evolution becomes qualitatively incorrect when $\left\langle\epsilon_{v_{e}}\right\rangle$ and $\left\langle\epsilon_{\bar{v}_{i}}\right\rangle$ surpass $\left\langle\epsilon_{v_{x}}\right\rangle$. Comparison with the results of Müller et al. (2012a) shows that this and the overall high predicted $\left\langle\epsilon_{v_{i}}\right\rangle$ are an artifact of the leakage/heating scheme.

(A color version of this figure is available in the online journal.)

(2012a) provide these luminosities from their 2D simulations in their Figure 8, we can directly compare with their results. Their $L_{v_{e}}$ peaks at $\sim 385 \mathrm{~B} \mathrm{~s}^{-1}\left(1 \mathrm{~B}=10^{51} \mathrm{erg}\right)$, while ours peaks at $\sim 365 \mathrm{~B} \mathrm{~s}^{-1}$ (a $5 \%$ difference). At $100 \mathrm{~ms}$ after bounce, the Müller et al. (2012a) simulation suggests $L_{v_{e}} \sim 62 \mathrm{~B} \mathrm{~s}^{-1}$, while we find $\sim 68 \mathrm{~B} \mathrm{~s}^{-1}$ in model $s 27 f_{\text {heat }} 1.15\left(\sim 73 \mathrm{~B} \mathrm{~s}^{-1}\right.$ in model $\left.s 27 f_{\text {heat }} 1.05\right), \mathrm{a} \sim 10 \%(\sim 20 \%)$ difference. The $\bar{v}_{e}$ and $v_{x}$ luminosities compare similarly well. The rather good agreement in total neutrino luminosities with the much more detailed radiation-hydrodynamics simulation of Müller et al. (2012a) suggests that our leakage/heating scheme captures the overall neutrino emission and its energetics in an acceptable way.

The situation is different for the mean neutrino energies $\left\langle\epsilon_{\nu}\right\rangle$ shown in the lower panel of Figure 8. We obtain estimates for $\left\langle\epsilon_{v}\right\rangle$ of each species by assuming blackbody emission from its neutrinosphere in the same way as for the mean-squared energies that enter the heating function (Equation (2)). This kind of estimate is not reliable in the very early, highly dynamical postbounce phase, but $\sim 20 \mathrm{~ms}$ after bounce, the usual hierarchy 
of neutrino energies $\left\langle\epsilon_{\nu_{x}}\right\rangle>\left\langle\epsilon_{\bar{v}_{e}}\right\rangle>\left\langle\epsilon_{v_{e}}\right\rangle$ is established analogously to the hierarchy of neutrino sphere radii (cf. lower panel of Figure 6). This hierarchy is, however, broken at times $\gtrsim 80-100 \mathrm{~ms}$, when the mean $\nu_{e}$ and $\bar{v}_{e}$ energies exceed the mean energy of $v_{x}$. This is clearly an artifact of our leakage/heating scheme and will not happen in nature. It is also not found by Müller et al. (2012a). The reason for this incorrect behavior can be understood by considering the neutrinosphere radii plotted in the lower panel of Figure 6 and looking at the temperature and $Y_{e}$ profiles shown in Figure 7 for model $s 27 f_{\text {heat }} 1.05$. At postbounce times $\gtrsim 80 \mathrm{~ms}$, one notices a global minimum in $Y_{e}$ around $40-50 \mathrm{~km}$. At the same location, a local temperature minimum develops. Both are related and caused by the inability of the leakage/heating scheme to establish a correct balance between emission and absorption at optical depths of a few. Unfortunately, the $v_{x}$ neutrinosphere recedes precisely into the local temperature minimum, while the $v_{e}$ and $\bar{v}_{e}$ neutrinospheres sit in the local maximum at slightly greater radii. While the differences in temperature are not large, they are sufficient to explain the incorrect evolution of the $\left\langle\epsilon_{v_{i}}\right\rangle$.

Comparing the values of $\left\langle\epsilon_{v_{i}}\right\rangle$ predicted by the leakage scheme with the results of Müller et al. (2012a) at times before the qualitative evolution becomes unreliable, we find that the leakage scheme systematically overpredicts the mean energies. For example, at $50 \mathrm{~ms}$ after bounce, in model $s 27 f_{\text {heat }} 1.05$, we find $\left\langle\epsilon_{v_{e}}\right\rangle \sim 16 \mathrm{MeV},\left\langle\epsilon_{\bar{v}_{e}}\right\rangle \sim 18 \mathrm{MeV}$, and $\left\langle\epsilon_{v_{x}}\right\rangle \sim 18.5 \mathrm{MeV}$. At the same time the $\left\langle\epsilon_{v_{i}}\right\rangle$ found by Müller et al. (2012a) are, in the same order, $9.3 \mathrm{MeV}, 12.3 \mathrm{MeV}$, and $14 \mathrm{MeV}$.

In summary, the results shown in this section indicate that the leakage/heating scheme used in our simulations yields overall qualitatively correct thermodynamics/stratification in the postshock region and captures the integral neutrino emission to within $\sim 20 \%$ of fully self-consistent simulations. It fails, however, to yield reliable predictions for the mean neutrino energies, in particular at later postbounce times. Since energy (and lepton number) absorption rates depend sensitively on neutrino energy, the leakage/heating scheme, at least in its present form, cannot be employed to make reliable predictions of the spectrum of the emitted neutrinos or the composition of explosion ejecta.

\subsection{SASI and Neutrino-driven Convection}

The recent $2 \mathrm{D}$ radiation-hydrodynamics core collapse and postbounce simulations of the $s 27$ progenitor carried out by Müller et al. (2012a) show a very clear and clean growth of a dominant periodic $\ell=1$ SASI mode. The relative amplitude (with respect to the average shock radius) of the $\ell=1$ mode saturates in their simulations at a very large $\sim 45 \%$, indicating a large-scale periodic dipole deformation. In their simulation, neutrino driven convection is only a secondary instability that develops in the nonlinear phase, but may be connected with the saturation itself (Guilet et al. 2010).

It is now interesting to ask if the SASI is the primary instability driving asphericity in the $s 27$ progenitor also in our 3D simulations or if neutrino-driven convection dominates early on and possibly suppresses the growth of coherent SASI oscillations. It is furthermore interesting to study how the roles and prominence of SASI and convection depend on the strength of neutrino heating. It is evident from the discussion in Section 3.1 and Figures 4 and 5 that deviations from sphericity develop at large scales in our models. We shall now take a more quantitative look at the development of this asphericity.

\subsubsection{Convection}

The local stability of a fluid element to convective overturn is determined via the Ledoux criterion (Ledoux 1947),

$$
C_{\mathrm{L}}=-\left(\frac{\partial \rho}{\partial P}\right)_{s, Y_{l}}\left[\left(\frac{\partial P}{\partial s}\right)_{\rho, Y_{l}}\left(\frac{d s}{d r}\right)+\left(\frac{\partial P}{\partial Y_{l}}\right)_{\rho, s}\left(\frac{d Y_{l}}{d r}\right)\right]
$$

which, in the postbounce supernova case, takes into account radial gradients in specific entropy $s$ and lepton fraction $Y_{l}=$ $Y_{e}+Y_{v_{e}}-Y_{\bar{v}_{e}}$. For simplicity, we set $Y_{l}:=Y_{e}$, since our leakage scheme does not keep track of local neutrino fractions. This approximation may lead to quantitatively incorrect estimates of $C_{\mathrm{L}}$ in the protoneutron star where neutrinos are trapped or partially trapped. A fluid element is convectively unstable if $C_{\mathrm{L}}>0$. The linear growth time for convection from arbitrarily small perturbations is then given by the Brunt-Väisälä (BV) frequency,

$$
\omega_{\mathrm{BV}}=\operatorname{sgn}\left(C_{\mathrm{L}}\right) \sqrt{\left|\frac{C_{\mathrm{L}}}{\rho} \frac{d \Phi}{d r}\right|},
$$

where we are following the definition of Buras et al. (2006a) and Takiwaki et al. (2012) and where $\Phi$ is the local gravitational potential and thus $d \Phi / d r$ is the local gravitational acceleration. For simplicity, we approximate the gravitational acceleration as $-G M(r) r^{-2}$ assuming an angle-averaged spherical matter distribution in our postprocessing analysis.

Foglizzo et al. (2006) pointed out that Equation (4) is an insufficient criterion for the development of large-scale convective instability in the postshock region. A small (linear) perturbation that could seed convection in the unstable gain layer is advected in toward the convectively stable cooling layer with the background flow. This advection may occur faster than the time it takes for convection to grow from the small perturbation. It is thus necessary to compare the advection timescale $\tau_{\text {adv }}$ with the growth time for convection in the gain layer, $\tau_{\mathrm{conv}} \approx \omega_{\mathrm{BV}}^{-1}$. Foglizzo et al. (2006) defined the quantity

$$
\chi=\int_{R_{\text {gain }}}^{R_{\text {shock }}} \frac{\omega_{\mathrm{BV}}}{\left|v_{r}\right|} d r=\frac{\tau_{\mathrm{adv}}}{\tau_{\mathrm{conv}}}
$$

where $v_{r}$ is the radial velocity through the gain region. A small-scale perturbation of magnitude $\delta_{\text {in }}$ entering the gain layer from above may at most grow by a factor $\exp (\chi)$ to $\delta_{\text {out }}=\delta_{\text {in }} \exp (\chi)$ during its advection through the gain layer (Scheck et al. 2008). According to the linear analysis of Foglizzo et al. (2006), $\chi \gtrsim 3$ is required for convection to develop in the gain layer from small perturbations $\delta_{\text {in }}$. Scheck et al. (2008) noted, then demonstrated, that the situation is different if the seed perturbations $\delta_{\text {in }}$ are sufficiently large so that the time integral of the buoyant acceleration becomes comparable to the advection velocity. In this case, the advected seed may grow into a buoyant plume and stay in the gain layer instead of leaving it. The results of Scheck et al. (2008) indicate that local seed perturbations of order $1 \%$, e.g., in the upstream radial velocity, may already be sufficient to trigger convection even if $\chi<3$.

If convection does develop, a simple measure of its strength is the anisotropic velocity $v_{\text {aniso, }}$, which we define, following Takiwaki et al. (2012), as

$$
v_{\text {aniso }}=\sqrt{\frac{\left\langle\rho\left[\left(v_{r}-\left\langle v_{r}\right\rangle_{4 \pi}\right)^{2}+v_{\theta}^{2}+v_{\varphi}^{2}\right]\right\rangle_{4 \pi}}{\langle\rho\rangle_{4 \pi}}},
$$



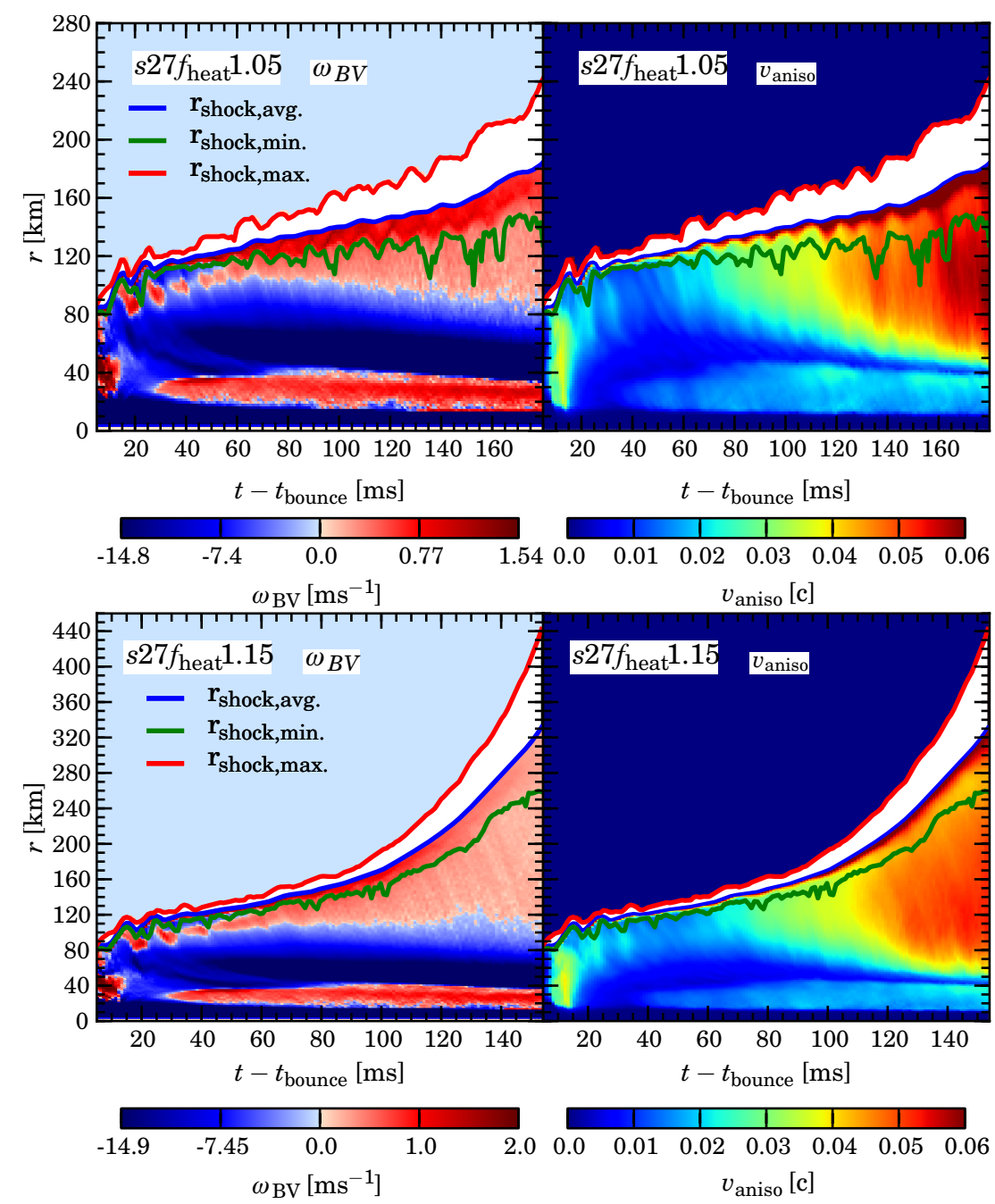

Figure 9. Color maps showing the time evolution of the angle-averaged Brunt-Väisälä (BV) frequency $\omega_{\mathrm{BV}}$ in units of ms ${ }^{-1}$ (Equation (5); left panels) and anisotropic velocity $v_{\text {aniso }}$ in units of the speed of light $c$ (Equation (7); right panels) in models $s 27 f_{\text {heat }} 1.05$ (top panels) and $s 27 f_{\text {heat }} 1.15$ (bottom panels). Also indicated are the maximum shock radius (red curves), the average shock radius (blue curves), and the minimum shock radius (green curves). Note the different radial scales of the top and bottom panels. We mask out $v_{\text {aniso }}$ and $\omega_{\mathrm{BV}}$ outside the average shock radius, where they are not reliable, since at most angles the radial region is actually outside of the shock. Shortly after bounce, the stalling shock leaves behind a negative entropy gradient, leading to $\omega_{\mathrm{BV}}>0$ and thus convective instability, strongest at radii between 20 and $40 \mathrm{~km}$. Prompt convection develops quickly and is strong, as indicated by the large $v_{\text {aniso }}$ in the right panels. Subsequently, convective instability and, as shown by the right panels, convection, develops in the gain layer and, after $\sim 30-40 \mathrm{~ms}$ or so, also at the edge of the protoneutron star core, due to the negative lepton gradient. Note that high $v_{\text {aniso }}$ at late times prevails to significantly smaller radii than the inner radius of convective instability, indicating large asymmetries and undershooting of decelerating convective plumes.

(A color version of this figure is available in the online journal.)

where $\langle.\rangle_{4 \pi}$ denotes an angle average at fixed radius. $v_{\text {aniso }}$ essentially extracts the magnitude of the velocity component that does not belong to a purely radial background flow. We compute $v_{\text {aniso }}$ by introducing a spherical auxiliary grid onto which we interpolate the Cartesian coordinate velocity components and transform to obtain $v_{r}, v_{\theta}$, and $v_{\varphi}$. We then integrate over $4 \pi$ steradian at each radius $r$ to obtain the various angleaveraged quantities. $v_{\text {aniso }}$ is high in regions of large fluctuations in $v_{r}$ and high non-radial velocities $v_{\theta}$ and $v_{\varphi}$. We note that high $v_{\text {aniso }}$ in the postshock region is a good measure for non-radial flow in that region. If this non-radial flow is due to prompt/neutrino-driven convection or induced by the SASI is difficult to decide, in particular when the SASI has reached the nonlinear regime. $v_{\text {aniso }}$ is thus most useful at early postbounce times and both its time evolution and radial distribution must be carefully considered.
In Figure 9, we present color maps showing the time evolutions of radial profiles of the angle-averaged $\omega_{\mathrm{BV}}$ (left panels) and $v_{\text {aniso }}$ (right panels) for models $s 27 f_{\text {heat }} 1.05$ (top panels) and $s 27 f_{\text {heat }} 1.15$ (bottom panels) as representative cases for moderate and strong neutrino heating. The qualitative evolution is the same in all models.

Within milliseconds of bounce, a highly convectively unstable region develops where the negative entropy gradient left behind by the stalling shock is strongest. As is evident from the $v_{\text {aniso }}$ color maps, a strong burst of prompt convection develops and smoothes out this entropy gradient within $\sim 20 \mathrm{~ms}$ of bounce. The highly dynamical early phase of shock expansion and prompt convection is over by $\sim 40 \mathrm{~ms}$ after bounce, when the shock has settled at $\sim 100-120 \mathrm{~km}$. At this time, the gain layer has developed and neutrino heating creates a negative entropy gradient and thus instability to convection between 


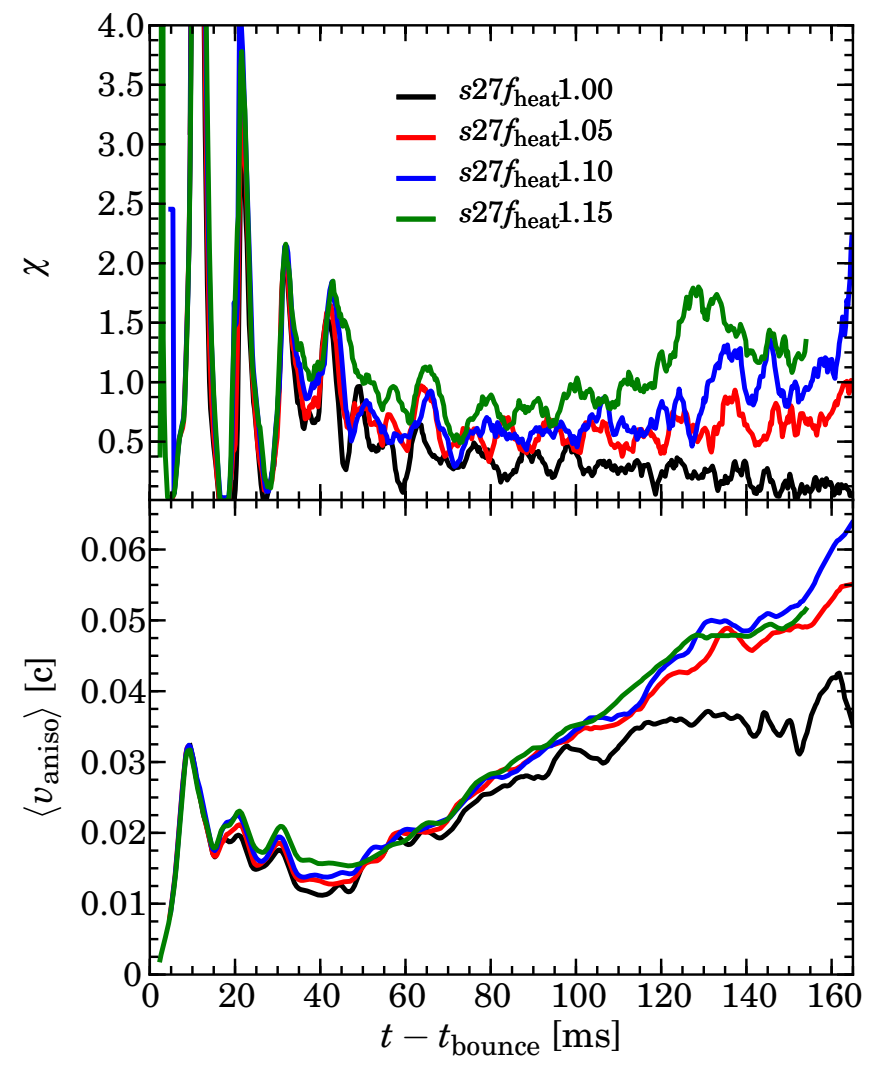

Figure 10. Top panel: Foglizzo parameter $\chi$ (Equation (6)) as a function of time after bounce. At times $\lesssim 40 \mathrm{~ms}$ after bounce, the shock is still expanding and a quasi-stationary gain layer has not yet developed. $\chi$ is not reliable in that phase. At later times, it stays consistently below the critical value of 3 suggested by Foglizzo et al. (2006) as being necessary for convection to develop from arbitrarily small perturbations. Note that stronger neutrino heating leads to greater $\chi$, since $\omega_{\mathrm{BV}}$ is larger. Bottom panel: density-weighted average of the anisotropic velocity $\left\langle v_{\text {aniso }}\right\rangle$ (Equation (7)) in the gain layer inside the minimum shock radius. As in the case of $\chi$, this quantity is not reliable in the highly dynamic early postbounce phase. The early peak around $10 \mathrm{~ms}$ is related to prompt convection, which ebbs over $\sim 30 \mathrm{~ms}$. Starting at $\sim 40 \mathrm{~ms}$ after bounce, when neutrino driving becomes efficient (cf. Figure 3), $\left\langle v_{\text {aniso }}\right\rangle$ increases nearly monotonically in a very similar way in all models. Only model $s 27 f_{\text {heat }} 1.00$, which has the weakest neutrino heating, deviates from this trend at times $\gtrsim 100 \mathrm{~ms}$ after bounce.

(A color version of this figure is available in the online journal.)

$\sim 80 \mathrm{~km}$ and the shock. Also, deleptonization at the edge of the protoneutron star (cf. Figure 7) creates a negative lepton gradient, driving protoneutron star convection, which sets in at 35-40 ms after bounce and is clearly marked by a band of high $v_{\text {aniso }}$, spatially coinciding with the band of convective instability in the protoneutron star.

Figure 10 shows the evolution of the Foglizzo parameter $\chi$ (Equation (6); top panel) and the density-weighted average anisotropic velocity in the gain layer (bottom panel). At the early postbounce times at which prompt convection takes place, both quantities are poorly defined, since the shock expansion is still rather dynamic and a quasi-stationary gain layer does not yet exist. This explains the large variations seen at early times in particular in $\chi$. Once the postbounce quasi-equilibrium in the postshock region is established, $\chi$ settles at values between 0 and 2 in all models, which is consistent with what Müller et al. (2012a) found for the $s 27$ progenitor.

The $\chi \gtrsim 3$ criterion proposed by Foglizzo et al. (2006) for the development of convection in the gain layer is never fulfilled in any of our models. Nevertheless, neutrino-driven convection does develop and becomes strong in all of our models. This is obvious from the radial $v_{\text {aniso }}$ distribution shown in Figure 9. As soon as the gain layer develops and $\omega_{\mathrm{BV}}$ becomes large, a broad region of high $v_{\text {aniso }}$ appears and traces the region of instability. This is indeed neutrino-driven convection, as can be seen from the entropy slices in the top panel of Figure 5, which show fully developed neutrino-driven convection at $\sim 80 \mathrm{~ms}$ after bounce. The development of neutrino-driven convection in the gain layer can also be inferred from the density-weighted average $v_{\text {aniso }}$ over the gain layer (bottom panel of Figure 10). $\left\langle v_{\text {aniso }}\right\rangle$ has an initial local maximum due to prompt convection and decreases as the latter ebbs only to increase again at $\gtrsim 40 \mathrm{~ms}$ after bounce when neutrino heating in the gain layer becomes efficient and drives convection (cf. Figure 3).

Müller et al. (2012a), in their axisymmetric simulation of the $s 27$ progenitor, did not observe the development of neutrino-driven convection, in agreement with the prediction of Foglizzo et al. (2006) that for $\chi \lesssim 3$ small perturbations are advected out of the gain layer before they can grow into buoyant plumes. While there are many technical differences between the simulation of Müller et al. (2012a) and the ones presented here, the key difference relevant for the development of neutrino-driven convection in our simulations is our choice of a Cartesian AMR grid as opposed to the spherical polar grid of the axisymmetric code of Müller et al. (2012a). A spherical polar grid is ideal for tracking the spherically-symmetric collapse phase and the upstream flow outside the shock after bounce. Seed perturbations remain minimal and neither prompt nor neutrino-driven convection grow in the simulation of Müller et al. (2012a). Our Cartesian AMR grid, on the other hand, leads to significant perturbations in multiple ways: (1) The Cartesian grid itself only imperfectly resolves spherical flow and perturbations of at most order $d x / R_{\text {shock }}$, where $d x$ is one linear computational cell size, are generated locally at the shock front. (2) Also due to its rectangular nature, the grid has $\ell=4, m=4$ symmetry, which leads to buildup of numerical noise primarily in modes with $\ell=4, m=\{-4,0,4\}$. (3) In our AMR setup, the shock is formed on the finest grid and then is allowed to pass through two mesh refinement boundaries before it reaches the grid that will track its subsequent evolution. The crossing of AMR boundaries causes large perturbations in the shock front that are also of $\ell=4$ character. (4) The AMR grid that tracks the shock front expands whenever the shock expands. The AMR boundary must constantly be filled via interpolation from the next coarser grid, which also introduces noise. Points (1) and (2) are true for any code using a Cartesian grid (e.g., the CASTRO code used in the recent simulations of Burrows et al. 2012; Murphy et al. 2012; Dolence et al. 2013), while (3) and (4) are due to our particular approach in Zelmani, which may or may not be different from what is done in CASTRO and other codes.

There are multiple ways in which one could quantify the magnitude of the perturbations present in the early postbounce phase in our models. One indicator may be the relative deviation of the shock front from spherical symmetry quantified in Figure 11. The $\ell=4$ grid modes indeed imprint themselves on the shock front, though the deviation of the shock itself from sphericity is not large. The root-square-sum $A_{4}$ of the normalized $\ell=4, m=\{-4, \ldots, 4\}$ components of the shock front has a maximum of $\sim 1.4 \%$ at $\sim 10 \mathrm{~ms}$ after bounce. This could be interpreted as a lower bound on the deviation from sphericity of the postshock flow and may already be sufficient to seed 


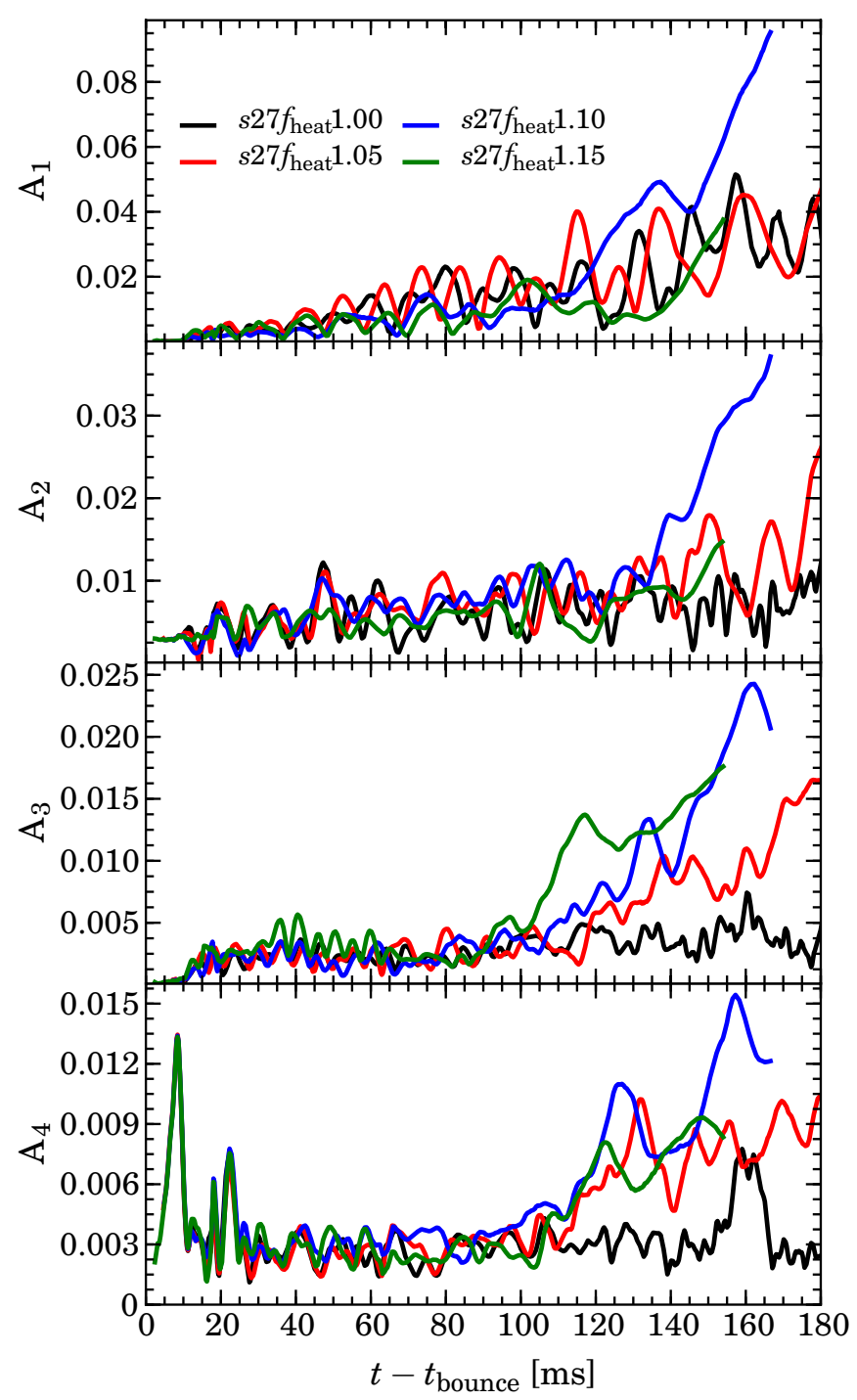

Figure 11. Time evolution of the normalized root-square-summed spherical harmonic mode amplitudes of the shock in all models for each $\ell$ in $\{1,2,3,4\}$ : $A_{1}, A_{2}, A_{3}$, and $A_{4}$ (Equation (11)). Note the dominance of the $\ell=4$ perturbations from the Cartesian grid at early times. The $A_{1}$ amplitude becomes dominant $\sim 40-50 \mathrm{~ms}$ after bounce and shows oscillatory features in models $s 27 f_{\text {heat }} 1.00$ and $s 27 f_{\text {heat }} 1.05$. Models $s 27 f_{\text {heat }} 1.10$ and $s 27 f_{\text {heat }} 1.15$ have no obvious oscillatory behavior of $A_{1}$, but develop large non-oscillatory amplitudes at late times when the shock in these models reaches large radii.

(A color version of this figure is available in the online journal.)

convection (Scheck et al. 2008). Alternatively, we consider the relative root-mean-square deviation from sphericity of any fluid quantity $X$ on a spherical shell of radius $R$,

$$
\xi(X)=\frac{\sqrt{\left\langle\left(X-\langle X\rangle_{4 \pi}\right)^{2}\right\rangle_{4 \pi}}}{\langle X\rangle_{4 \pi}},
$$

where $\langle\cdot\rangle_{4 \pi}$ denotes an angular average at fixed radius and we have dropped the dependence on $R$ for simplicity. Evaluating $\xi$ for density, radial velocity, entropy, and pressure in the preshock region $\left(R>R_{\text {shock, } \max }\right.$ ), we find only very small deviations from sphericity of order $0.1 \%$ at any time. We carry out this analysis also at a radius just inside the shock (dynamically adjusting $R$ to be $\sim R_{\text {shock,min }}-1 \mathrm{~km}$ ), which should be reliable in the dynamical shock expansion phase. Any perturbations would have to come from shock passage, since convection had no time to grow. For this, we find large deviations of 5\%-10\% in density, entropy, and pressure. ${ }^{15}$ These deviations are present already milliseconds after bounce and they peak when the shock passes through the boundary of the second finest refinement level (at $59 \mathrm{~km}$ ) at $\sim 3 \mathrm{~ms}$ after bounce. This indicates that shock passage through refinement boundaries may be the dominant source of numerical perturbations in our simulations.

The large-amplitude perturbations present in the early postbounce flow are more than sufficient to overcome advection and seed prompt convection, which grows within milliseconds of bounce in our models (cf. Figures 9 and 10). Neutrino-driven convection is, in turn, seeded by the turbulent flow of prompt convection and by additional, though much smaller magnitude, noise coming from interpolation at the AMR boundary and from the Cartesian representation of the spherically accreting outer core.

$$
\text { 3.3.2. SASI }
$$

Convective overturn, first prompt, then neutrino-driven, develops early on in our simulations and appears dominant. We can, however, not yet exclude growth of the SASI. The conditions for SASI growth are very different from those for convection. Any standing accretion shock is unstable to the SASI, with $\ell=1, m=0, \pm 1$ modes being the most unstable and growing from arbitrarily small perturbations (e.g., Guilet \& Foglizzo 2012). The linear growth rate of the SASI can be expressed as

$$
\omega_{\mathrm{SASI}}=\frac{\ln |\mathcal{Q}|}{\tau_{\mathrm{cyc}}},
$$

where $\mathcal{Q}$ is the cycle efficiency, defined as the amplification factor of perturbations in each advective-acoustic cycle, and $\tau_{\text {cyc }}$ is the duration of a cycle (see, e.g., Scheck et al. 2008 for a detailed discussion). Qualitatively, $\tau_{\text {cyc }}$ depends on the radius at which the shock stalls and on the timescale for advection of entropy/vorticity perturbations between shock and protoneutron star edge. A smaller shock radius and shorter advection time will thus lead to a smaller $\tau_{\text {cyc }}$ and faster SASI growth. Strong neutrino heating, as pointed out by Yamasaki \& Yamada (2007) and Scheck et al. (2008), increases the buoyancy in the gain layer and leads to both larger $\mathcal{Q}$ and shock oscillation frequencies (connected with $\tau_{\text {cyc }}$ ), while the growth rate is not strongly affected.

A characteristic feature of the SASI in its linear phase is the exponential growth of oscillatory low-mode deformations of the shock front. We look for evidence for the SASI in our simulations by decomposing the shock surface $R_{\text {shock }}(\theta, \phi)$ into spherical harmonics:

$$
a_{\ell m}=\frac{(-1)^{|m|}}{\sqrt{4 \pi(2 \ell+1)}} \int_{4 \pi} R_{\text {shock }}(\theta, \phi) Y_{\ell}^{m}(\theta, \phi) d \Omega .
$$

Note that $a_{00}$ corresponds to the average shock radius and that the definition of the $a_{\ell m}$ used here gives individual $a_{\ell m}$ amplitudes that are a factor of $(2 \ell+1)$ smaller than the definition for $a_{\ell 0}$ used by Müller et al. (2012a) in the axisymmetric case, but at each $\ell$, there are $(2 \ell+1)$ more modes in our case. The $Y_{\ell}^{m}$ are the standard real spherical harmonics (e.g., Boas 2006), which we use with the normalization factors given in Burrows et al. (2012). We also define the quantities $A_{\ell}$ as the root-square-sum

\footnotetext{
15 The deviation of the radial velocity is of order unity there, which is readily explained by the extreme variation of $v_{r}$ across the shock and is thus not a reliable measure.
} 

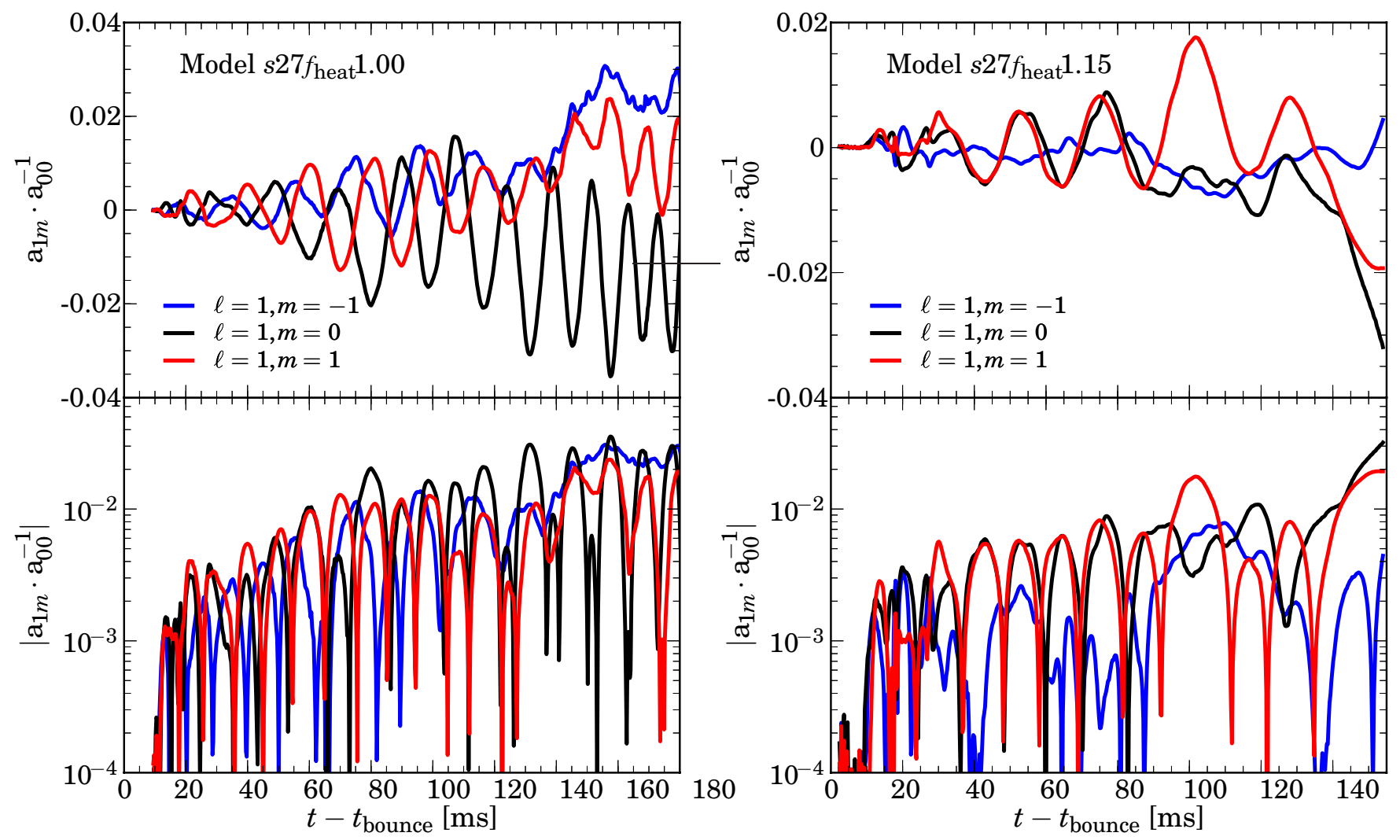

Figure 12. Normalized $\ell=1, m=\{-1,0,1\}$ mode amplitudes $a_{1 m} / a_{00}$ of the shock front plotted on a linear scale (top panels) and their absolute values plotted on a logarithmic scale (bottom panels) for models $s 27 f_{\text {heat }} 1.00$ (left panels) and $s 27 f_{\text {heat }} 1.15$ (right panels). Model $s 27 f_{\text {heat }} 1.00$ shows a clear exponential growth of oscillatory modes, but saturation occurs at amplitudes that are about an order of magnitude smaller than in the 2D simulation of the same progenitor carried out by Müller et al. (2012a). Model $s 27 f_{\text {heat }} 1.15$, which has strong neutrino heating and intense neutrino-driven convection also shows some oscillatory $\ell=1$ mode growth, though at a longer oscillation period, lower saturation amplitudes, and without a well defined exponential growth phase.

(A color version of this figure is available in the online journal.)

of the $a_{\ell m}$ for a given $\ell$ normalized by the average shock radius $a_{00}$,

$$
A_{\ell}=\frac{1}{a_{00}} \sqrt{\sum_{m=-\ell}^{\ell} a_{\ell m}^{2}} .
$$

In Figure 11, we present in four panels, from top to bottom, the time evolutions of the $A_{1}-A_{4}$ amplitudes of the shock front in all four models. In the first $\sim 20 \mathrm{~ms}$ after bounce, the initial $\ell=4$ deformation due to our Cartesian grid imprints itself onto the shock front and the $A_{4}$ amplitude is dominant. Subsequently, the other modes grow. For SASI growth, the expectation is that the $\ell=1, m=\{-1,0,1\}$ modes have the fastest growth rate and have oscillatory behavior, which should be reflected in the $A_{1}$ amplitude. In models $s 27 f_{\text {heat }} 1.00$ and $s 27 f_{\text {heat }} 1.05, A_{1}$ indeed is the fastest growing amplitude and shows the expected oscillatory behavior throughout the simulated postbounce interval, suggesting the presence of the SASI. However, the maximum value of $A_{1}$ reached is $\sim 0.04$, which is an order of magnitude smaller than what was reported by Müller et al. (2012a) for their 2D simulation of the $s 27$ progenitor.

For the two models with stronger neutrino heating, $s 27 f_{\text {heat }} 1.10$ and $s 27 f_{\text {heat }} 1.15$, the situation is different. Their $A_{1}$ and $A_{2}$ amplitudes hover around very similar small values without obvious oscillatory behavior until $\sim 100 \mathrm{~ms}$ after bounce, when large-scale deviations from sphericity (cf. Figure 4) lead to strongly growing amplitudes in all $\ell$. This was also observed in the high-luminosity light-bulb simulations of Burrows et al. (2012) and Dolence et al. (2013). $A_{1}$ is the dominant amplitude and reaches $\sim 0.1$ in model $s 27 f_{\text {heat }} 1.10$ and about 0.03 in model $s 27 f_{\text {heat }} 1.15$ at the end of its simulation. It is interesting to note that model $s 27 f_{\text {heat }} 1.05$, which has a positively trending shock radius at the end of its simulation, has clearly growing $A_{2}, A_{3}$, and $A_{4}$ amplitudes at late times, while $A_{1}$ remains the dominant mode with stable amplitudes near 0.03 .

For further insight into the nature of the observed mode evolution, we plot, in Figure 12, the individual $\ell=1, m=\{-1,0,1\}$ normalized mode amplitudes $a_{1 m} / a_{00}$ in linear (top panels) and logarithmic scale (bottom panels) for models $s 27 f_{\text {heat }} 1.00$ (left panels) and $s 27 f_{\text {heat }} 1.15$ (right panels). The former model has the weakest neutrino heating and least vigorous neutrino-driven convection of all our models while the latter model has the strongest heating and most vigorous convection. All $\ell=1$ modes in model $s 27 f_{\text {heat }} 1.00$ show a clear oscillatory behavior and, importantly, an exponential growth phase between $\sim 20$ and $\sim 80 \mathrm{~ms}$ after bounce can be made out. However, saturation occurs at low $a_{1 m} / a_{00} \sim 0.01$ for all modes. As noted before, this is an order of magnitude smaller than found in the axisymmetric simulations of Müller et al. (2012a; in which neutrino-driven convection did not develop as a primary instability).

Interestingly, some of the $a_{1 m} / a_{00}$ modes in model $s 27 f_{\text {heat }} 1.15$ do exhibit oscillatory behavior, though with larger periods than in model $s 27 f_{\text {heat }} 1.00$. This is expected for SASI growth under the influence of strong neutrino heating (Yamasaki \& Yamada 2007; Scheck et al. 2008). The growth also saturates 
more quickly at amplitudes that remain a factor of $\sim 2$ smaller than in model $s 27 f_{\text {heat }} 1.00$ until $\sim 100 \mathrm{~ms}$ after bounce, when the mode growth becomes non-oscillatory. It is not possible to unambiguously and clearly identify a phase of exponential growth of the $a_{1 m} / a_{00}$ modes in this model.

In summary, there is clear evidence for SASI growth in our models. It is strongest in the model with the least neutrino heating and weakest neutrino-driven convection. It is weakest in the model with the most neutrino heating and the strongest neutrinodriven convection. However, even in the model in which SASI growth is strongest, the SASI saturates at amplitudes that are an order of magnitude smaller than in the 2D simulation of Müller et al. (2012a), which did not have any neutrino-driven convection. These observations suggest that $3 \mathrm{D}$ neutrino-driven convection is indeed detrimental to the development of largeamplitude SASI. This confirms the findings of Scheck et al. (2008), Guilet et al. (2010), Burrows et al. (2012), and Dolence et al. (2013). Furthermore, our results show that both instabilities can coexist and grow at the same time, but even if convection is suppressed (a case we cannot study in our 3D Cartesian AMR code), the nearly equal splitting of the $\ell=1$ power across the three azimuthal $m$ modes in $3 \mathrm{D}$, will likely reduce the magnitude of deviations from sphericity that can be driven by the SASI alone. Moreover, the SASI, once it has reached its nonlinear phase, will trigger neutrino-driven convection (Scheck et al. 2008; Guilet et al. 2010; Burrows et al. 2012; Müller et al. 2012a), which may very well become the dominant instability, in particular if neutrino heating is strong.

\subsection{Criteria for Neutrino-driven Explosions}

The simulations presented here end before an explosion is fully developed in any of our models. Nevertheless, interesting trends can be observed. Models $s 27 f_{\text {heat }} 1.10$ and $s 27 f_{\text {heat }} 1.15$ have strongly positively trending shock radii at the end of their simulations. The shock in model $s 27 f_{\text {heat }} 1.05$ also expands at late times, but the development of an explosion is definitely more marginal. Model $s 27 f_{\text {heat }} 1.00$ has a receding shock and thus a rather negative prognosis regarding explosion.

A variety of criteria for neutrino-driven explosions have been discussed in the literature and it is interesting to see how the trends observed in our models compare with what is expected from theory and other simulation results.

From the bottom panel of Figure 3 we find that models with stronger neutrino heating and, thus, more vigorous neutrinodriven convection have systematically more mass in the gain layer $\left(M_{\text {gain }}\right)$ that can absorb neutrino energy. The low-amplitude SASI seen in our models, which is strongest in models with weakest heating and convection, does not appear to have any positive effect on $M_{\text {gain }}$ in our simulations. In models that are trending toward explosion, $M_{\text {gain }}$ increases as shock expansion sets in. This is consistent with previous work giving the most optimistic prognosis for models with the greatest $M_{\text {gain }}$ (e.g., Murphy \& Burrows 2008; Scheck et al. 2008; Müller et al. 2012b; Hanke et al. 2012).

Also shown in the bottom panel of Figure 3 is the densityweighted average of the specific entropy in the gain layer $\left(\left\langle s_{\text {gain }}\right\rangle\right)$. All models, trending toward explosion or not, exhibit the same $\left\langle s_{\text {gain }}\right\rangle$ evolution until $\sim 130 \mathrm{~ms}$ after bounce, when the most optimistic models actually move to somewhat smaller $\left\langle s_{\text {gain }}\right\rangle$ (cf. the discussion in Section 3.1). Thus, in agreement with Hanke et al. (2012), the average entropy in the gain layer is not a good indicator for a model's potential for explosion.
A criterion frequently used to diagnose neutrino-driven explosions arises from the comparison of the timescale for neutrino heating $\tau_{\text {heat }}$ and the advection timescale $\tau_{\text {adv }}$ for material to pass through the gain layer (Burrows \& Goshy 1993; Janka 2001; Thompson et al. 2005; Murphy \& Burrows 2008). If heating is faster than advection through the gain layer, then a fluid parcel entering the gain region may absorb sufficient energy to reach positive total specific energy and thus become unbound. For $\tau_{\text {adv }} / \tau_{\text {heat }} \gtrsim 1$, shock expansion should set in, further increasing $\tau_{\text {adv }}$ and thus leading to positive feedback and runaway expansion.

In our simplified analysis, we set $\tau_{\text {heat }}=\left|E_{\text {gain }}\right| / Q_{\text {net }}$, where $Q_{\text {net }}$ is the net integral heating rate in the gain layer and $\left|E_{\text {gain }}\right|$ is the volume integral of the (Newtonian) total specific energy of material in the gain layer, given, e.g., by the integral over Equation (3) of Müller et al. (2012b). We note that the internal energy of the LS220 EOS is defined with respect to a free neutron gas, this defines the zero of our internal energy. There are a variety of possible definitions for $\tau_{\mathrm{adv}}$ (cf. the discussions in Murphy \& Burrows 2008; Marek \& Janka 2009; Müller et al. $2012 b$ ). Here, we use the definition $\tau_{\text {adv }}=\dot{M} / M_{\text {gain }}$, where $M_{\text {gain }}$ is the mass in the gain region and $\dot{M}$ is the accretion rate through the shock. Note that this definition is different from what we use in the computation of the Foglizzo $\chi$ parameter (Equation (6)).

In the top panel of Figure 13, we plot $\tau_{\text {adv }} / \tau_{\text {heat }}$ as a function of time after bounce for all of our models (left ordinate). The behavior is as expected: the two models $s 27 f_{\text {heat }} 1.15$ and $s 27 f_{\text {heat }} 1.10$, which are strongly trending toward explosion reach $\tau_{\text {adv }} / \tau_{\text {heat }} \gtrsim 1$ already at $\sim 100$ and $\sim 115 \mathrm{~ms}$ after bounce. The marginal model $s 27 f_{\text {heat }} 1.05$ also shows increasing $\tau_{\text {adv }} / \tau_{\text {heat }}$, which reaches 1 at $\sim 142 \mathrm{~ms}$ after bounce. There is, however, no hope for model $s 27 f_{\text {heat }} 1.00$, where $\tau_{\text {adv }} / \tau_{\text {heat }}$ always remains below $\sim 0.5$.

Also shown in the top panel of Figure 13 is $E_{\text {pos }}$ (right ordinate), the integral energy of unbound material (with positive total specific energy, again defining the internal energy with respect to a free neutron gas). When $\tau_{\text {adv }} / \tau_{\text {heat }}>1.4$ in our models, material starts to become unbound and $E_{\text {pos }}$ grows rapidly. However, at the end of our simulations, it is still far away from the energy needed to unbind the entire envelope and lead to a canonical $\sim 1 B$ core-collapse supernova explosion. We caution the reader to not overinterpret $E_{\text {pos }}$-it is unreliable at this point. Rather, what is important to note is that toward the end of the simulations there is an increasing amount of unbound material for the highest values of $f_{\text {heat }}$. To obtain a quantitatively reliable measure of the asymptotic explosion energy one must follow the explosion to late times, consistently track or account for recombination $(\sim 8-9 \mathrm{MeV}$ per nucleon), and consider the binding energy of the overlying envelope $(\sim 1 \mathrm{~B}$; Woosley et al. 2002).

Finally, in the bottom panel of Figure 13, we plot the time evolution of the maximum of the ratio of the angleaveraged square of the speed of sound $\left\langle c_{s}^{2}\right\rangle$ to the angleaveraged square of the escape velocity, which we approximate as $\left\langle v_{\text {esc }}^{2}\right\rangle \approx 2 G M(r) / r$, where $M(r)$ is the enclosed baryonic mass. This ratio is interesting, since Pejcha \& Thompson (2012) have recently derived the antesonic condition,

$$
\max \left(\frac{c_{s}^{2}}{v_{\text {esc }}^{2}}\right)>\frac{3}{16} \approx 0.19,
$$

beyond which no solution for a stationary spherically symmetric accretion shock exists, marking the transition to explosion. 


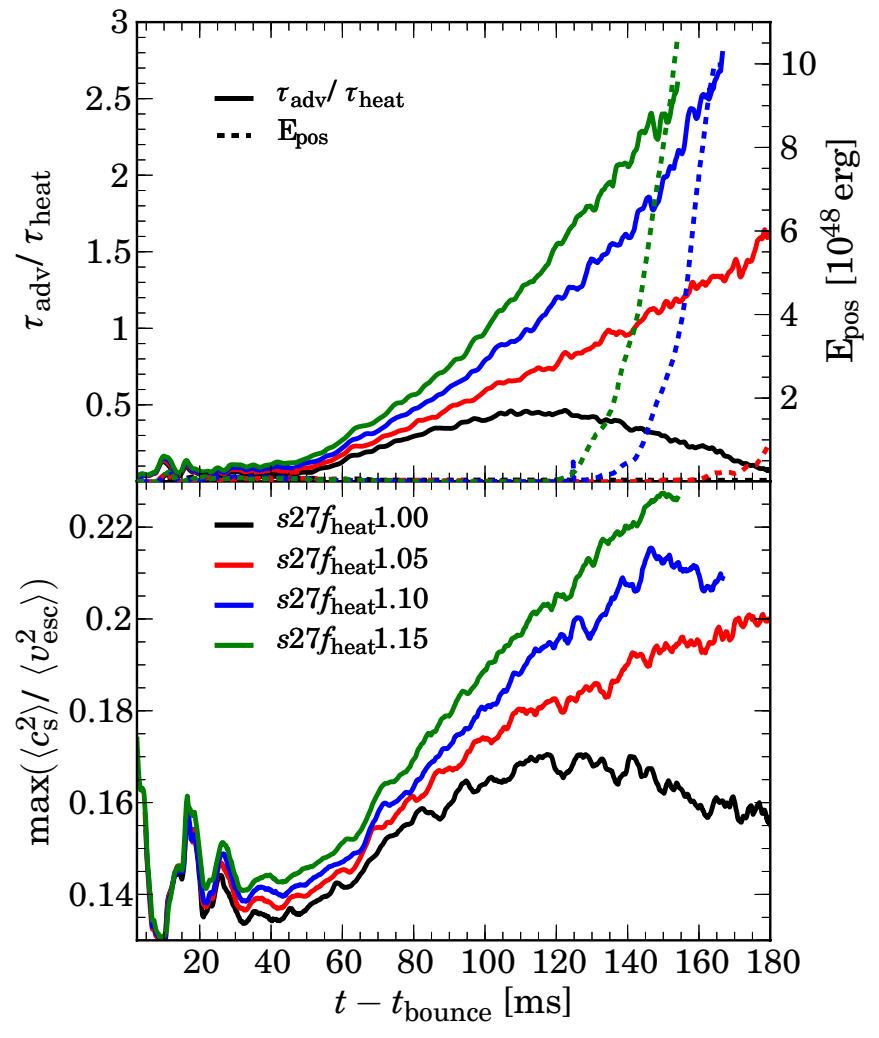

Figure 13. Top panel: ratio of advection and heating timescales $\tau_{\mathrm{adv}} / \tau_{\text {heat }}$ as a function of time after bounce in our models (left ordinate) and $E_{\text {pos }}$, the volume integral over positive values of the total specific energy in the gain region (right ordinate). $\tau_{\text {adv }} / \tau_{\text {heat }} \gtrsim 1$ is considered to be a condition for runaway explosion. It is satisfied by all of our models with optimistic outlook. Models $s 27 f_{\text {heat }} 1.15$, $s 27 f_{\text {heat }} 1.10$, and $s 27 f_{\text {heat }} 1.15$ reach the threshold at $\sim 100, \sim 115$, and $\sim 142 \mathrm{~ms}$ after bounce, respectively. Roughly $\sim 20 \mathrm{~ms}$ later, these models are beginning to develop regions with positive total energy, which may be interpreted as the onset of explosion. Bottom panel: maximum of the ratio of the angle-averaged squared speed of sound to the angle-averaged squared escape velocity. According to the antesonic condition of Pejcha \& Thompson (2012), no solution for a spherical stationary accretion shock exists for $\max \left(c_{s}^{2} / v_{\mathrm{esc}}^{2}\right)>3 / 16 \approx 0.19$ and an explosion is expected to set in models that surpass this value.

(A color version of this figure is available in the online journal.)

While the expanding shocks in our models are far away from sphericity, we find values of $\max \left(\left\langle c_{\mathrm{s}}^{2}\right\rangle /\left\langle v_{\mathrm{esc}}^{2}\right\rangle\right) \gtrsim 0.2-0.22$, which is consistent with the expectation of Pejcha \& Thompson (2012). Model $s 27 f_{\text {heat }} 1.00$, which has the most pessimistic outlook, does not reach $\max \left(\left\langle c_{\mathrm{s}}^{2}\right\rangle /\left\langle v_{\text {esc }}^{2}\right\rangle\right) \gtrsim 0.19$, while the marginal model, $s 27 f_{\text {heat }} 1.05$ does. The prognosis, according to Pejcha \& Thompson (2012) is thus similar to the one based on the $\tau_{\text {adv }} / \tau_{\text {heat }}>1$ runaway condition.

\subsection{Gravitational Wave Signals}

Besides the neutrino signals already discussed in Section 3.2, GWs are the only other direct probe of the processes occurring in the postshock region and in the protoneutron star. The overall GW signature of core-collapse supernovae has been reviewed in detail by Ott (2009) and Kotake (2011) and we refer the interested reader to these reviews for an in-depth discussion of the various potential GW emission processes and their underlying physics.

GW observations of the next galactic core-collapse supernova could provide important insight into the role and relevance of multi-dimensional fluid instabilities, rotation, the structure of the protoneutron star, and the nuclear EOS (Dimmelmeier et al.
2008; Marek et al. 2009; Yakunin et al. 2010; Murphy et al. 2009; Röver et al. 2009; Ott 2009; Kotake 2011). Recently, Logue et al. (2012) carried out a proof-of-principle study, demonstrating that Bayesian inference allows to select between different explosion mechanisms for a galactic core-collapse supernova. The reliability of this depends on the availability of robust waveform predictions from simulations. Most currently available core-collapse supernova waveforms come from 2D simulations (as summarized by Ott 2009; Kotake et al. 2011), which can predict only one of the two independent polarizations. In the context of nonrotating or slowly rotating neutrino-driven corecollapse supernovae, only very few waveform predictions from 3D simulations without symmetry constraints exist. Fryer et al. (2004), carried out Newtonian 3D smoothed-particle hydrodynamics simulations with gray flux-limited diffusion neutrino transport and studied the GW emission from matter motions and asymmetric neutrino emission up to $\sim 80 \mathrm{~ms}$ after bounce in a variety of different precollapse configurations with and without initial rotation and large-scale asphericities. Kotake et al. (2009, 2011) performed Newtonian 3D hydrodynamic simulations with a light-bulb scheme (similar to MB08, but with a better approximation to changes in $Y_{e}$ ). They used analytic initial conditions, a fixed accretion rate and a fixed inner spherical boundary at $50 \mathrm{~km}$, but were able to evolve for $\sim 500 \mathrm{~ms}$ and studied the GW emission from matter dynamics and asymmetric neutrino emission. Scheidegger et al. (2010) performed full 3D Cartesian (without inner boundary) Newtonian collapse and postbounce simulations of a slowly rotating progenitor with neutrino leakage (but no heating). They employed a monopole approximation for gravity with relativistic corrections and evolved to $\sim 100 \mathrm{~ms}$ after bounce. Recently, Müller et al. (2012c) presented Newtonian $3 \mathrm{D}$ postbounce simulations with GR corrections to the monopole term of the Newtonian potential. They used a time-dependent inner boundary that contracts from $60-80 \mathrm{~km}$ to $15-25 \mathrm{~km}$ over $1 \mathrm{~s}$ following the prescription of Scheck et al. (2008), but were able to evolve multiple progenitor models for $\gtrsim 1.2 \mathrm{~s}$ using a ray-byray gray two-species approximate transport scheme (neglecting $v_{x}$ ) and imposed neutrino luminosities at the inner boundary. They extracted and studied in detail the GW emission due to matter dynamics and anisotropic neutrino emission.

While the simulations presented in this study do not have the more sophisticated neutrino transport treatment of Müller et al. (2012c), they do not have an artificial inner boundary with imposed core neutrino luminosities, are carried out in full GR, and include the cooling due to $v_{x}$ emission from the protoneutron star. It is, hence, worthwhile to study the GWs emitted by our models. We restrict ourselves to GWs from the dominant accelerated quadrupole matter motions and ignore GWs from asymmetric neutrino emission. The rationale for the latter is that our simple leakage scheme is unfit to give a reasonable estimate for the true neutrino radiation field anisotropy leading to GW emission. Moreover, as demonstrated by previous work (Kotake et al. 2009, 2011; Müller et al. 2012c; Marek et al. 2009; Yakunin et al. 2010), GW emission due to asymmetric neutrino emission occurs at too low frequencies to be relevant for earthbound detectors such as Advanced LIGO (for the LIGO Scientific Collaboration: Harry 2010; Shoemaker 2010), Advanced Virgo (Virgo Collaboration: Accadia et al. 2011), and KAGRA (for the KAGRA Collaboration: Somiya 2012).

We employ the quadrupole approximation for extracting GWs from our simulations and use the expressions detailed in Ott et al. (2012). In principle, we could extract the gravitational waveforms directly from the spacetime, but the results of 

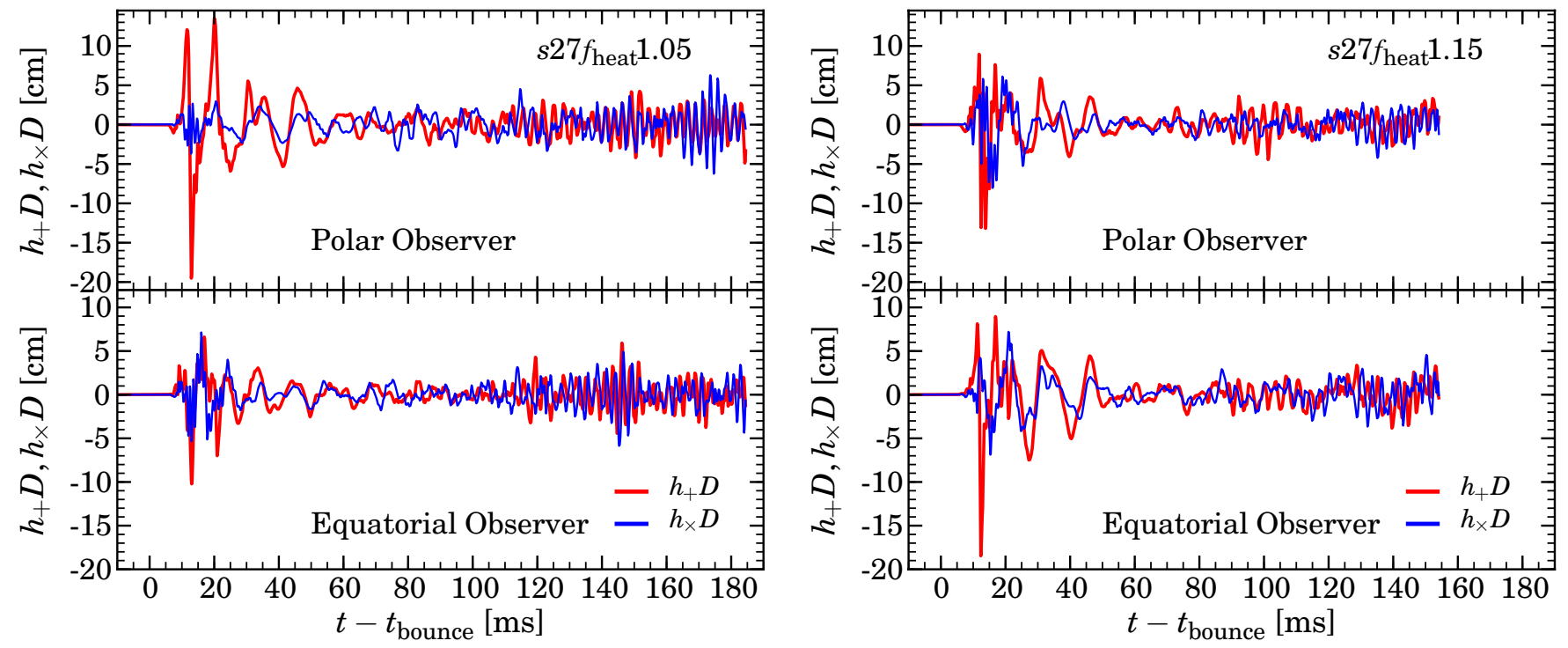

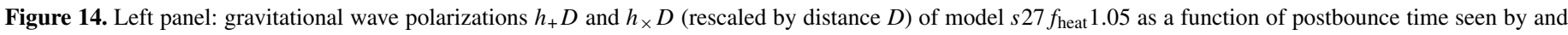

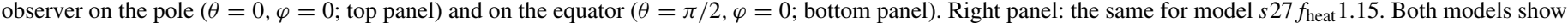

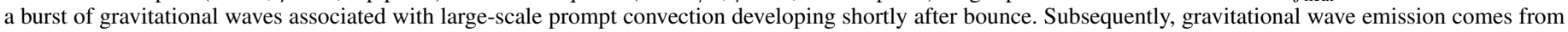

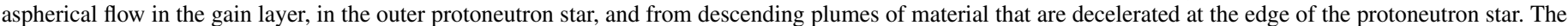
gravitational wave signals are trending toward higher frequencies with time.

(A color version of this figure is available in the online journal.)

Reisswig et al. (2011) suggest that the quadrupole approximation is very likely sufficiently accurate for stellar collapse spacetimes with a protoneutron star. The full observer-angle independent GW signals for all models are available for download from http://www.stellarcollapse.org/gwcatalog.

In Figure 14, we plot the $h_{+}$and $h_{\times}$polarizations of the GW signal (rescaled by distance $D$ ) for model $s 27 f_{\text {heat }} 1.05$ (left panel) and model $s 27 f_{\text {heat }} 1.15$ (right panel) as seen by observers on the north pole $(\theta=0, \varphi=0$; top panels) and on the equator $(\theta=\pi / 2, \varphi=0$; bottom panels). The GW signals emitted by the other models are very similar and not shown. The early emission sets in $\sim 10 \mathrm{~ms}$ after bounce and is due to prompt convection that dominates the aspherical dynamics in the early postbounce phase, but has decayed by $\sim 40 \mathrm{~ms}$ after bounce. The GW signal from convection and other fluid instabilities is of stochastic nature (cf. Kotake et al. 2009; Ott 2009) and its time series cannot be predicted exactly. The GW signal of prompt convection, since it is emitted within milliseconds of bounce by the strongest first few overturn cycles, is particular sensitive to the perturbations seeding prompt convection. Note that the time series of $h_{+}$and $h_{\times}$from prompt convection in the two models are quite different, but the overall amplitudes agree well, but peak in different viewing directions. The subsequent evolution of the GW signals is similar in both models, both polarizations, and both observer positions. After an intermittent quiescent phase, GW emission picks up again at times $\gtrsim 80 \mathrm{~ms}$ after bounce when aspherical dynamics becomes strong throughout the entire postshock region (cf. Figure 9). In this phase, the $\mathrm{GW}$ emission transitions to higher frequencies, indicating that emission from deceleration of downflows at the steep density gradient at the edge of the protoneutron star (as first pointed out by Murphy et al. 2009) and convection in the protoneutron star play an increasing role. While both models have expanding shocks at the end of their simulations, the shock acceleration has not become sufficiently strong to lead to an offset in the GW signal (GW memory) seen in other work that followed exploding models to later times (e.g., Murphy et al. 2009; Yakunin et al. 2010; Müller et al. 2012c; Kotake et al. 2009, 2011).

The peak GW strain amplitudes reached in our models are from prompt convection and go up to $|h| D \sim 20 \mathrm{~cm}$ $\left(\sim 6.5 \times 10^{22}\right.$ at $\left.10 \mathrm{kpc}\right)$. Scheidegger et al. (2010) found $|h| D \sim 10 \mathrm{~cm}$ and Fryer et al. (2004) found $|h| D \sim 12 \mathrm{~cm}$, but we note that the GW signal will depend on the strength of prompt convection, which is different from model to model. The approaches of Müller et al. (2012c) and Kotake et al. $(2009,2011)$ do not allow them to study prompt convection. The typical amplitudes reached in the pre-explosion phase are $\sim 3 \mathrm{~cm}\left(\sim 10^{-22}\right.$ at $\left.10 \mathrm{kpc}\right)$. This is comparable to, but somewhat larger than what Müller et al. (2012c) found in the pre-explosion phase of their models. This may be due the different progenitor models used and/or to the rather large inner boundary radius of their models in the pre-explosion phase. Our typical $|h|$ are also quantitatively consistent with the findings of the simpler 3D simulations of Scheidegger et al. (2010) and Kotake et al. $(2009,2011)$, but are a factor of a few smaller than predictions from 2D simulations (e.g., Marek et al. 2009; Yakunin et al. 2010; Murphy et al. 2009).

Figure 15 contrasts the angle-averaged characteristic GW strain spectra $h_{\text {char }}(f)$ (Flanagan \& Hughes 1998) of our models with the broadband design noise levels of advanced-generation GW interferometers, assuming a source distance of $10 \mathrm{kpc}$. The spectra are scaled with a factor of $f^{-1 / 2}$ to allow one-toone comparison with the detector one-sided amplitude spectral noise density $\sqrt{S(f)}$, which has units of $\mathrm{Hz}^{1 / 2}$. Most of the detectable emission is within $\sim 60-1000 \mathrm{~Hz}$ and at essentially the same level of $\sim 2-6 \times 10^{-23} \mathrm{~Hz}^{-1 / 2}$. A galactic event (at $10 \mathrm{kpc}$ ) appears to be well detectable by the upcoming generation of detectors. All four models, while having distinct individual $h_{+}$and $h_{\times}$time series that vary greatly in the time domain, exhibit essentially the same robust spectral features, 


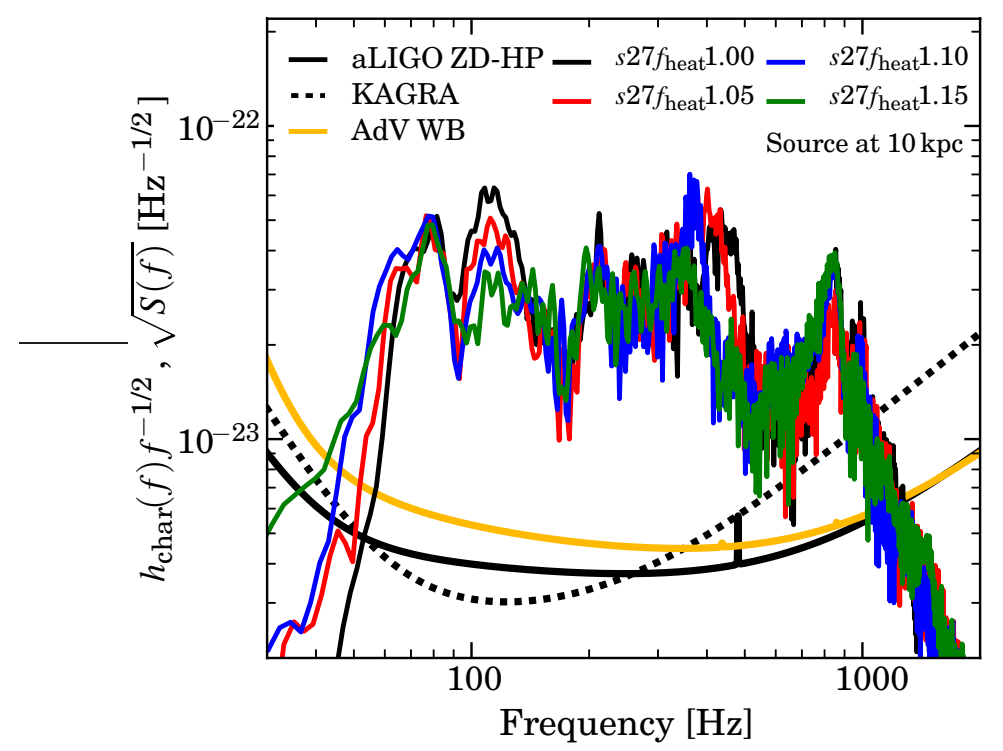

Figure 15. Characteristic spectral strain spectra $h_{\text {char }}(f) f^{-1 / 2}$ of all four models at a distance of $10 \mathrm{kpc}$ compared with the design noise levels $\sqrt{S(f)}$ of Advanced LIGO in the broadband zero-detuning high-power mode (aLIGO ZD-HP), KAGRA, and Advanced Virgo in wideband mode (AdV WB).

(A color version of this figure is available in the online journal.)

independent of $f_{\text {heat }}$ and the exact postbounce time the individual models are evolved to. The low-frequency to intermediatefrequency emission is most likely due to prompt convection in the early postbounce phase, while the high-frequency peaks at $\sim 400 \mathrm{~Hz}$ and $\sim 900 \mathrm{~Hz}$ are most likely due to the deceleration of downflows at the protoneutron star surface and protoneutron star convection. A more detailed investigation of these features must be left to future work, since it would require multiple quadrupole integrals to isolate emission regions as done, e.g., by Murphy et al. (2009). Our present simulations provide only one global quadrupole integral and we do not have sufficiently finely sampled output for a postprocessing analysis of the GW signal.

The total energy emitted in GWs is $3-4 \times 10^{-10} M_{\odot} c^{2}$ in all our models and about $50 \%$ of the emitted energy is due to the higher-frequency GW emission at later postbounce times. This finding is consistent with the 3D results of Scheidegger et al. (2010). Müller et al. (2012c), on the other hand, found emitted GW energies of only $\sim 10^{-11} M_{\odot} c^{2}$. Their models do not include prompt convection and emit most of their GW energy at frequencies below $\sim 400-600 \mathrm{~Hz}$. This, again, may be due to the different considered progenitor structures and/or to the inner boundary of their simulations.

\section{DISCUSSION AND CONCLUSIONS}

We have carried out four 3D GR core collapse and postbounce simulations of the $27 M_{\odot}$ solar-metallicity progenitor of Woosley et al. (2002), systematically varying the rate of neutrino energy deposition to study the effect of variations in neutrino heating on the 3D postbounce evolution in general and on the SASI in particular. These simulations neither employed an artificial inner boundary nor did they make any symmetry assumptions or approximations for the gravitational field. The resolution of our simulations is nearly twice as high and we carried them out for nearly twice as long as the only previous 3D GR study of Kuroda et al. (2012).

For neutrinos, we used an energy-averaged (gray) threespecies neutrino leakage/heating scheme in the postbounce phase, whose only free parameter is a scaling factor in the energy deposition rate. The leakage scheme captures the essential aspects of neutrino cooling, lepton number exchange, and neutrino heating as predicted by fully self-consistent $1 \mathrm{D}$ and 2D neutrino radiation-hydrodynamics simulations. Importantly, our simulations do not suffer from the limitations of simpler analytic "light-bulb" heating/cooling schemes, which cannot capture the contraction and deleptonization of the protoneutron star and result in artificially large shock radii and overestimated advection times through the postshock region (S. Richers et al. 2013, in preparation). The light-bulb approach, due to its simplicity and low computational cost, is being employed in many contemporary 3D simulations (e.g., Nordhaus et al. 2010; Hanke et al. 2012; Burrows et al. 2012; Murphy et al. 2012; Dolence et al. 2013). However, as pointed out by Hanke et al. (2012) and Müller et al. (2012a), light-bulb calculations may yield qualitatively incorrect results for the postbounce hydrodynamics and the respective roles and relevance of neutrino-driven convection and the SASI.

Our approach was designed specifically to avoid the problems of the light-bulb scheme and provide a realistic postbounce setting for more robust conclusions on the postbounce evolution and the role of hydrodynamic instabilities. At the same time, our leakage/scheme is still computationally much cheaper and simpler than the approximate gray or energy-dependent 3D neutrino transport schemes of Kuroda et al. (2012), Takiwaki et al. (2012), Müller et al. (2012c), and Wongwathanarat et al. (2010). This affords us with the ability to carry out parameter studies with high numerical resolution as presented in this work for the $27 M_{\odot}$ progenitor.

Müller et al. (2012a) previously carried out an axisymmetric (2D) simulation of the same $27 M_{\odot}$ progenitor with their 2D GR radiation-hydrodynamics code. They found neutrinodriven convection to be suppressed due to the high postbounce accretion rate and, thus, short advection time through the convectively unstable gain layer. The SASI is the primary instability in their simulation and seeds convection, which grows only as a secondary instability once the SASI has reached nonlinear amplitudes.

Our models show instead early and strong growth of convective instability. It is initially prompt, driven by the negative 
entropy gradient left behind by the stalling shock. Subsequently, convection is driven by neutrino energy deposition in the gain layer. Neutrino-driven convection first manifests itself in smallscale local rising hotter and sinking cooler blobs of postshock material. In models with strong neutrino heating that are trending toward explosion, the small-scale blobs combine over time to a few large, near volume-filling high-entropy regions whose expansion pushes out the shock. This was also observed in the high-luminosity light-bulb simulations of Burrows et al. (2012) and Dolence et al. (2013). These large blobs lead to a low- $\ell-$ mode dominated structure of the expanding shock. The shock, however, has a complicated substructure of protruding bumps caused by smaller-scale plumes that perturb it locally. Models whose shock expansion becomes dynamical, surpass the runaway explosion criterion $\tau_{\text {adv }} / \tau_{\text {heat }} \gtrsim 1$ (Burrows \& Goshy 1993; Janka 2001) and satisfy the antesonic condition of Pejcha \& Thompson (2012). Both criteria for explosion yield predictions consistent with the trends in our models. Interestingly, shortly after the $\tau_{\text {adv }} / \tau_{\text {heat }} \gtrsim 1$ condition is met by our models, individual fluid cells behind the shock reach positive total energy, indicating the transition to explosion.

While neutrino-driven convection is the fastest growing and overall dominant instability, our analysis suggests that all of our models exhibit some growth of clearly periodic low- $\ell$ deformations of the shock front that are characteristic of the linear phase of the SASI. As expected from linear perturbation analysis, we find that the $\ell=1, m=\{-1,0,1\}$ modes exhibit the fastest growth. However, our results also show that the saturation amplitudes of the oscillatory $\ell=1, m=\{-1,0,1\}$ modes are, in the best case, an order of magnitude smaller than in Müller et al. (2012a). The SASI remains a sub-dominant instability in all of our models. Furthermore, we find the SASI to be strongest in the model with the least neutrino heating and the weakest neutrino-driven convection. Models with stronger heating and more vigorous convection have lower saturation amplitudes of the oscillatory modes, but develop large non-oscillatory deformations of $\ell=1,2,3$ character that are caused by low-mode neutrino-driven convection and are unrelated to the SASI.

Our simulations satisfy all the requirements laid out by Müller et al. (2012a) for the development of strong SASI in the $27 M_{\odot}$ progenitor: GR gravity, an EOS that results in a fairly compact protoneutron star, and the inclusion of all neutrino species and deleptonization of the protoneutron star. Yet, our results turn out to be very different from what Müller et al. (2012a) found. What is the root cause of this discrepancy? On the one hand, our simulations are $3 \mathrm{D}$, splitting, on average, the $2 \mathrm{D}$ $\ell=1$ SASI power across three azimuthal $m$ modes. This may explain lower saturation amplitudes, but cannot explain the early growth of neutrino-driven convection that is absent from the 2D simulation of Müller et al. (2012a). On the other hand-as we are convinced, more importantly-our simulations used a central Cartesian AMR grid, which imparts perturbations of order of $1 \%-10 \%$ onto the very early postbounce flow, seeding prompt convection. This, in turn, acts as seed for neutrinodriven convection in our models. The seed perturbations are sufficiently large for convection to develop despite the high accretion rate and correspondingly short advection time through the gain layer. Neutrino-driven convection becomes dominant and limits the growth of the SASI, in agreement with the 2D work of Scheck et al. (2008). We expect any 3D simulation relying on 3D Cartesian AMR with similar resolution to have similarly large seed perturbations for convection. The recent
3D light-bulb simulations of Burrows et al. (2012), Murphy et al. (2012), and Dolence et al. (2013) are all subject to these perturbations.

The question of the magnitude of seed perturbations was not raised by Müller et al. (2012a), who used a spherical-polar grid that leads to only minute perturbations from the growth of numerical noise during collapse. Is the almost perfectly spherical postbounce state of Müller et al. (2012a) representative of nature or should one expect significant asphericities to be present in the outer core? Some guidance on the size of perturbations induced by turbulent convection during late time burning in core-collapse supernova progenitors is already available from the 2D and 3D simulations of Meakin, Arnett, and collaborators (Meakin 2006; Meakin \& Arnett 2006, 2007b; Arnett \& Meakin 2011).

There are two important results from these multi-dimensional stellar evolution calculations that pertain to the expected density perturbation amplitudes in precollapse cores. First, 2D and 3D simulations of the oxygen shell burning dominated phase in a $23 M_{\odot}$ star (Meakin \& Arnett 2007b) have clarified the basic mechanism responsible for the origin of the fluctuations. In short, Meakin \& Arnett (2007a) found that the root-meansquare (rms) density fluctuations are largest at the convective boundaries. By interpreting the dynamics of the convective boundary layer in terms of $g$-modes excited by the turbulent convection, it was shown that the rms density fluctuation amplitude can be related directly to the background stellar structure and the Mach number of the convective flow, with

$$
\frac{\delta \rho}{\rho} \sim M_{c}^{2}+\frac{v_{s} \omega_{\mathrm{BV}} M_{c}}{g},
$$

where $M_{c}$ is the rms Mach number of the convective flow, $\omega_{\mathrm{BV}}$ is the Brunt-Väisälä frequency in the stable layer adjacent to the convection zone, $v_{s}$ is the sound speed of the gas, and $g$ is the gravitational acceleration. The first term on the righthand side, which is very small, is relevant to the interior of the convection zone, where density fluctuations arise solely from the presence of velocity fluctuations in a nearly adiabatic layer. The second term is significantly larger and applies to the stable layers bounding the convection zone, reflecting the excitation of fluid motions in these regions in the form of internal waves (predominantly $g$-modes).

The $27 M_{\odot}$ progenitor of Woosley et al. 2002 has a turbulent Mach number of $\sim 0.1$ to 0.2 in the silicon burning convective shell overlying the core, and two peaks in $\omega_{\mathrm{BV}}$ of importance: the peak corresponding to the inner edge of the active silicon burning shell (corresponding to the outer edge of the iron core), and a peak deeper in associated with the outermost extent of the now extinguished silicon burning core. Both peaks have values of $v_{s} \omega_{\mathrm{BV}} / g$ of $\sim 1$, indicating that rms density fluctuations at these locations will be of order the turbulence Mach number of the convection, or $\sim 10 \%-20 \%$. The spike in $\omega_{\mathrm{BV}}$ associated with the outer extent of the silicon core burning epoch will be accreted into the shock within $\sim 15$ ms of bounce, while the edge of the iron core will be accreted a little later, at $\sim 60 \mathrm{~ms}$ after bounce.

The second result from the multi-dimensional stellar convection simulations of Meakin and Arnett involves the interaction of nuclear burning shells at late times. While the results on boundary layer fluctuations described above are considered to be robust by those authors, the presence of two or more convective shells in close proximity, as found in late burnings stages, 
has been found to drive additional motion at the convective boundaries and correspondingly larger density fluctuation amplitudes. In the most relevant case of a silicon burning shell around an iron core, the interaction between the silicon, oxygen, neon, and carbon shells were found to produce a dramatic increase in boundary layer distortion, eventually leading to a complete disruption and mixing of the multi-shell burning region (Meakin 2006; Arnett \& Meakin 2011). This result is likely to be due, at least in part, to the inconsistency between the initial stellar model used (based on mixing length theory) and a more realistic turbulent convection as represented by the numerical simulation. Judging the robustness of these shell-interaction results, however, awaits 3D simulations since all of the multi-shell calculations performed to date have been restricted to 2D geometry which is known to result in exaggerated velocities in regions of thermal convection. From this body of work, it would appear that the presence of density fluctuations with amplitudes of at least $1 \%$, and possibly as large as $10 \%-20 \%$, should be expected in the material accreting into the shock at early postbounce times in a collapsing iron core.

The fast growth of neutrino-driven convection in our current models is almost certainly caused by the large seed perturbations from our Cartesian AMR grid. In 2D simulations, the growth of neutrino-driven convection may go along with SASI growth or, if not genuine SASI, then at least large-scale oscillatory low- $\ell$ deformations of the shock front (Müller et al. 2012a; Burrows et al. 2012; Fernández \& Thompson 2009a; Scheck et al. 2008). Our 3D models do not exhibit any large-scale oscillatory features. Rather, models evolving toward an explosion develop non-oscillatory large-scale asphericities at late times and produce a globally aspherical explosion morphology without a need for SASI-driven $\ell=1$ deformations. This qualitative finding is in agreement with the results of the convection-dominated 3D Newtonian light-bulb calculations of Burrows et al. (2012) and Dolence et al. (2013). The late-time development of SASI-like oscillatory behavior seen in 2D simulations that are initially convection dominated (e.g., Marek \& Janka 2009; Müller et al. 2012b) may thus be an artifact of axisymmetry, but further work is required to solidify this conclusion.

The next galactic core-collapse supernova will reveal its inner workings by means of its neutrino and GW signals. Both will provide key insight into the thermodynamics and multidimensional dynamics of the protoneutron star and the postshock region (e.g., Ott 2009; Lund et al. 2010, 2012; O'Connor \& Ott 2013). While our neutrino treatment is too simplistic to yield quantitatively interesting predictions of the neutrino signal, we are in a good position to study the GW emission from accelerated quadrupole mass motions in our models: For the first time, we have extracted GWs from full 3D GR collapse and postbounce core-collapse supernova simulations. We find a strong burst of GWs associated with early-postbounce prompt convection with frequencies around $\sim 100-200 \mathrm{~Hz}$, a subsequent almost quiescent phase, followed by higher-frequency $(400-1000 \mathrm{~Hz})$ emission, whose amplitudes are dominated by the deceleration of undershooting convective plumes at the edge of the protoneutron star (cf. Murphy et al. 2009). If convection (prompt and/or neutrino-driven) does not develop early, the GW signal would not have a strong initial burst, but rather a slow rise to smaller amplitudes at later times, when the SASI becomes strong. This is a key difference and may allow GW data analysts to distinguish between convection-dominated and SASI-dominated postbounce evolution in the next galactic core-collapse supernova. The design sensitivities of advanced-generation $\mathrm{GW}$ de- tectors such as Advanced LIGO, Advanced Virgo, or KAGRA are likely to be sufficient to detect the collapse and neutrinodriven explosion in our $27 M_{\odot}$ progenitor throughout the Milky Way. While different in detail, our results for the GW signature are generally consistent with what was found for other progenitors in the 2D first-principles simulations of Marek et al. (2009) and Yakunin et al. (2010). Our GW signals have higher amplitudes and characteristic frequencies than predicted by the 3D simulations of Müller et al. (2012c), who employed an artificial inner boundary that was moved in according to an analytic prescription.

There are a number of shortcomings and limitations of the simulations presented here that must be mentioned and can be removed only by future work. As is well known and has been pointed out recently by Hanke et al. (2012) in the core-collapse context, in 3D, turbulent power cascades to small scales. Low resolution in 3D may artificially keep power at large scales and may thus lead to an overestimate of the positive effect of neutrino-driven convection. While our effective angular and radial resolution in the postshock gain layer is comparable to the highest resolution considered by Hanke et al. (2012), we agree with their assessment that understanding the resolution dependence of 3D results is of great importance. We will carry out a resolution study in future work.

The second major limitation of our simulations is our Cartesian AMR grid and the fact that we must let the nascent supernova shock pass two mesh refinement boundaries before tracking its further evolution by AMR. This induces large perturbations leading to the growth of prompt and neutrino-driven convection in all of our models. These large and essentially unavoidable seed perturbations for prompt and neutrino-driven convection make it difficult to draw conclusions on which hydrodynamic instability dominates in the early postbounce phase. This limitation is shared by other Cartesian AMR schemes. It could possibly be avoided in future work by extending our spherical-polar grid blocks all the way into the protoneutron star core and using a single high-resolution Cartesian mesh only in the innermost few kilometers. Also in future work, we intend to carry out a study in which we map a postbounce profile from a 1D collapse simulation onto our 3D grid after the shock has passed the radii of the inner refinement levels. This should allow us to investigate the role of seed perturbations in a more controlled way.

A third major limitation of our work is the reliance on our simple gray heating/leakage scheme. While superior to the light-bulb approach, it cannot replace the energy-dependent neutrino radiation-hydrodynamics treatment that has proven to be crucial for reliable conclusions on the neutrino mechanism (e.g., Müller et al. 2012b and references therein). The set of 3D GR hydrodynamics simulations presented here required about $\sim 20$ million CPU hours to complete. Adding energydependent 3D neutrino transport will increase the computational complexity by an order of magnitude. Novel, highly efficient and scalable approaches to 3D neutrino transport will be needed to address this problem (Sumiyoshi \& Yamada 2012; Abdikamalov et al. 2012; Zhang et al. 2013; Radice et al. 2012).

We acknowledge helpful discussions with Dave Arnett, Adam Burrows, Sean Couch, Luc Dessart, Thierry Foglizzo, Uschi C. T. Gamma, Sarah Gossan, Raph Hix, H.-Thomas Janka, Peter Kalmus, Hannah Klion, Io Kleiser, Jim Lattimer, Bernhard Müller, Jeremiah Murphy, David Radice, Luke Roberts, Jason Nordhaus, Ken Nomoto, Jerome Novak, Tony 
Piro, Sherwood Richers, and members of our Simulating eXtreme Spacetimes (SXS) collaboration (http://www.blackholes.org). This research is partially supported by NSF grant Nos. AST-0855535, AST-1212170, PHY-0904015, PHY-1151197, OCI-0905046, and OCI-0941653, by the Sloan Research Foundation, and by the Sherman Fairchild Foundation. C.R. acknowledges support by NASA through Einstein Postdoctoral Fellowship grant No. PF2-130099 awarded by the Chandra X-ray center, which is operated by the Smithsonian Astrophysical Observatory for NASA under contract NAS8-03060. R.H. acknowledges support by the Natural Sciences and Engineering Council of Canada. The simulations were performed on the Caltech compute cluster "Zwicky" (NSF MRI award No. PHY-0960291), on supercomputers of the NSF XSEDE network under computer time allocation TG-PHY100033, on machines of the Louisiana Optical Network Initiative under grant loni_numrel07, and at the National Energy Research Scientific Computing Center (NERSC), which is supported by the Office of Science of the US Department of Energy under contract DE-AC02-05CH11231. The multi-dimensional visualizations were generated with the open-source VisIt visualization package (https://wci.llnl.gov/codes/visit/). All other figures were generated with the Python-based matplotlib package (http://matplotlib.org/).

\section{REFERENCES}

Abdikamalov, E., Burrows, A., Ott, C. D., et al. 2012, ApJ, 755, 111

Accadia, T., Acernese, F., Antonucci, F., et al. (Virgo Collaboration) 2011, CQGra, 28, 114002

Alcubierre, M., Brügmann, B., Diener, P., et al. 2003, PhRvD, 67, 084023

Alcubierre, M., Brügmann, B., Dramlitsch, T., et al. 2000, PhRvD, 62, 044034

Arnett, W. D., \& Meakin, C. 2011, ApJ, 733, 78

Baade, W., \& Zwicky, F. 1934, PNAS, 20, 254

Baiotti, B., Hawke, I., Montero, P. J., et al. 2005, PhRvD, 71, 024035

Baumgarte, T. W., Montero, P. J., Cordero-Carrión, I., \& Müller, E. 2013, PhRvD, 87, 044026

Baumgarte, T. W., \& Shapiro, S. L. 1999, PhRvD, 59, 024007

Baumgarte, T. W., \& Shapiro, S. L. 2010, Numerical Relativity: Solving Einstein's Equations on the Computer (Cambridge: Cambridge Univ. Press)

Berger, M. J., \& Oliger, J. 1984, JChPh, 53, 484

Bethe, H. A. 1990, RvMP, 62, 801

Bethe, H. A., \& Wilson, J. R. 1985, ApJ, 295, 14

Bionta, R. M., Blewitt, G., Bratton, C. B., Casper, D., \& Ciocio, A. 1987, PhRvL, 58,1494

Blondin, J. M., \& Mezzacappa, A. 2007, Natur, 445, 58

Blondin, J. M., Mezzacappa, A., \& DeMarino, C. 2003, ApJ, 584, 971

Boas, M. L. 2006, Mathematical Methods in the Physical Sciences (3rd ed.; Hoboken, NJ: Wiley)

Brandt, T. D., Burrows, A., Ott, C. D., \& Livne, E. 2011, ApJ, 728, 8

Buras, R., Janka, H.-T., Rampp, M., \& Kifonidis, K. 2006a, A\&A, 457, 281

Buras, R., Rampp, M., Janka, H.-T., \& Kifonidis, K. 2006b, A\&A, 447, 1049

Burrows, A., Dessart, L., \& Livne, E. 2007a, in AIP Conf. Ser. 937, Supernova 1987A: 20 Years After: Supernovae and Gamma-Ray Bursters, ed. S. Immler \& R. McCray (Melville, NY: AIP), 370

Burrows, A., Dessart, L., Livne, E., Ott, C. D., \& Murphy, J. 2007b, ApJ, 664,416

Burrows, A., Dolence, J. C., \& Murphy, J. W. 2012, ApJ, 759, 5

Burrows, A., \& Goshy, J. 1993, ApJL, 416, L75

Burrows, A., Hayes, J., \& Fryxell, B. A. 1995, ApJ, 450, 830

Chandrasekhar, S. 1961, Hydrodynamic and Hydromagnetic Stability (Oxford: Clarendon)

Dasgupta, B., Fischer, T., Horiuchi, S., et al. 2010, PhRvD, 81, 103005

Demorest, P. B., Pennucci, T., Ransom, S. M., Roberts, M. S. E., \& Hessels, J. W. T. 2010, Natur, 467, 1081

Dessart, L., Burrows, A., Livne, E., \& Ott, C. D. 2006, ApJ, 645, 534

Dimmelmeier, H., Ott, C. D., Marek, A., \& Janka, H.-T. 2008, PhRvD, 78, 064056

Dolence, J. C., Burrows, A., Murphy, J. W., \& Nordhaus, J. 2013, ApJ, 765, 110

Einfeldt, B. 1988, in Proceedings of the Sixteenth International Symposium, in Shock Tubes and Waves (Weinheim: VCH), 671
Fernández, R. 2010, ApJ, 725, 1563

Fernández, R., \& Thompson, C. 2009a, ApJ, 703, 1464

Fernández, R., \& Thompson, C. 2009b, ApJ, 697, 1827

Flanagan, É. É., \& Hughes, S. A. 1998, PhRvD, 57, 4535

Foglizzo, T. 2002, A\&A, 392, 353

Foglizzo, T., Galletti, P., Scheck, L., \& Janka, H.-T. 2007, ApJ, 654, 1006

Foglizzo, T., Scheck, L., \& Janka, H.-T. 2006, ApJ, 652, 1436

Font, J. A., Miller, M., Suen, W.-M., \& Tobias, M. 2000, PhRvD, 61, 044011

Fryer, C. L., \& Heger, A. 2000, ApJ, 541, 1033

Fryer, C. L., Holz, D. E., \& Hughes, S. A. 2004, ApJ, 609, 288

Goodale, T., Allen, G., Lanfermann, G., et al. 2003, in 5th International Conference, Vector and Parallel Processing_VECPAR'2002, ed. J. M. L. M. Palma, J. Dongarra, V. Hernández, A. A. Sousa, \& M. Waldén (Lecture Notes in Computer Science; Berlin: Springer)

Guilet, J., \& Foglizzo, T. 2012, MNRAS, 421, 546

Guilet, J., Sato, J., \& Foglizzo, T. 2010, ApJ, 713, 1350

Hanke, F., Marek, A., Müller, B., \& Janka, H.-T. 2012, ApJ, 755, 138

Harry, G. M. (for the LIGO Scientific Collaboration) 2010, CQGra, 27, 084006

Hebeler, K., Lattimer, J. M., Pethick, C. J., \& Schwenk, A. 2010, PhRvL, 105, 161102

Herant, M., Benz, W., \& Colgate, S. 1992, ApJ, 395, 642

Herant, M., Benz, W., Hix, W. R., Fryer, C. L., \& Colgate, S. A. 1994, ApJ, 435,339

Hirata, K., Kajita, T., Koshiba, M., Nakahata, M., \& Oyama, Y. 1987, PhRvL, 58,1490

Hüdepohl, L., Müller, B., Janka, H.-T., Marek, A., \& Raffelt, G. G. 2010, PhRvL, 104,251101

Hyman, J. M. 1976, The Method of Lines Solution of Partial Differential Equations, Tech. Rep., ERDA Mathematics and Computing Laboratory, Courant Institute of Mathematical Sciences, New York University

Iwakami, W., Kotake, K., Ohnishi, N., Yamada, S., \& Sawada, K. 2008, ApJ, 678,1207

Iwakami, W., Kotake, K., Ohnishi, N., Yamada, S., \& Sawada, K. 2009, ApJ, 700,232

Janka, H.-T. 2001, A\&A, 368, 527

Janka, H.-T., Langanke, K., Marek, A., Martínez-Pinedo, G., \& Müller, B. 2007, $\mathrm{PhR}, 442,38$

Janka, H.-T., \& Müller, E. 1995, ApJL, 448, L109

Janka, H.-T., \& Müller, E. 1996, A\&A, 306, 167

Kitaura, F. S., Janka, H.-T., \& Hillebrandt, W. 2006, A\&A, 450, 345

Kotake, K. 2011, Special Issue of Comptes Rendus Physique "Gravitational Waves (from Detectors to Astrophysics)," submitted (arXiv:1110.5107)

Kotake, K., Iwakami, W., Ohnishi, N., \& Yamada, S. 2009, ApJL, 697, L133

Kotake, K., Iwakami Nakano, W., \& Ohnishi, N. 2011, ApJ, 736, 124

Kuroda, T., Kotake, K., \& Takiwaki, T. 2012, ApJ, 755, 11

Lattimer, J. M., \& Prakash, M. 2001, ApJ, 550, 426

Lattimer, J. M., \& Swesty, F. D. 1991, NuPhA, 535, 331

Ledoux, P. 1947, ApJ, 105, 305

Lentz, E. J., Mezzacappa, A., Bronson Messer, O. E., et al. 2012, ApJ, 747, 73 Liebendörfer, M. 2005, ApJ, 633, 1042

Liebendörfer, M., Mezzacappa, A., \& Thielemann, F. 2001, PhRvD, 63, 104003

Löffler, F., Faber, J., Bentivegna, E., et al. 2012, CQGra, 29, 115001

Logue, J., Ott, C. D., Heng, I. S., Kalmus, P., \& Scargill, J. 2012, PhRvD, 86, 044023

Lund, T., Marek, A., Lunardini, C., Janka, H.-T., \& Raffelt, G. 2010, PhRvD, 82,063007

Lund, T., Wongwathanarat, A., Janka, H.-T., Müller, E., \& Raffelt, G. 2012, PhRvD, 86, 105031

Marek, A., \& Janka, H.-T. 2009, ApJ, 694, 664

Marek, A., Janka, H.-T., \& Müller, E. 2009, A\&A, 496, 475

McCorquodale, P., \& Colella, P. 2011, Commun. Appl. Math. Comput. Sci., 6,

Meakin, C. A. 2006, PhD thesis, The Univ. of Arizona, Tucson, Arizona

Meakin, C. A., \& Arnett, D. 2006, ApJL, 637, L53

Meakin, C. A., \& Arnett, D. 2007a, ApJ, 665, 690

Meakin, C. A., \& Arnett, D. 2007b, ApJ, 667, 448

Müller, B., Janka, H.-T., \& Dimmelmeier, H. 2010, ApJS, 189, 104

Müller, B., Janka, H.-T., \& Heger, A. 2012a, ApJ, 761, 72

Müller, B., Janka, H.-T., \& Marek, A. 2012b, ApJ, 756, 84

Müller, E., Janka, H.-T., \& Wongwathanarat, A. 2012c, A\&A, 537, A63

Murphy, J. W., \& Burrows, A. 2008, ApJ, 688, 1159

Murphy, J. W., Dolence, J. C., \& Burrows, A. 2012, ApJ, submitted (arXiv:1205.3491)

Murphy, J. W., Ott, C. D., \& Burrows, A. 2009, ApJ, 707, 1173

Nordhaus, J., Burrows, A., Almgren, A., \& Bell, J. 2010, ApJ, 720, 694

O'Connor, E., \& Ott, C. D. 2010, CQGra, 27, 114103

O'Connor, E., \& Ott, C. D. 2011, ApJ, 730, 70 
O’Connor, E., \& Ott, C. D. 2013, ApJ, 762, 126

Ohnishi, N., Kotake, K., \& Yamada, S. 2006, ApJ, 641, 1018

Ott, C. D. 2009, CQGra, 26, 063001

Ott, C. D., Abdikamalov, E., O’Connor, E., et al. 2012, PhRvD, 86, 024026

Ott, C. D., Burrows, A., Dessart, L., \& Livne, E. 2008, ApJ, 685, 1069

Ott, C. D., Dimmelmeier, H., Marek, A., et al. 2007a, PhRvL, 98, 261101

Ott, C. D., Dimmelmeier, H., Marek, A., et al. 2007b, CQGra, 24, 139

Pejcha, O., \& Thompson, T. A. 2012, ApJ, 746, 106

Pollney, D., Reisswig, C., Schnetter, E., Dorband, N., \& Diener, P. 2011, PhRvD, 83,044045

Radice, D., Abdikamalov, E., Rezzolla, L., \& Ott, C. D. 2012, JCoPh, 242, 648

Rampp, M., \& Janka, H.-T. 2002, A\&A, 396, 361

Rantsiou, E., Burrows, A., Nordhaus, J., \& Almgren, A. 2011, ApJ, 732, 57

Reisswig, C., Haas, R., Ott, C. D., et al. 2013, PhRvD, accepted (arXiv:1212.1191)

Reisswig, C., Ott, C. D., Sperhake, U., \& Schnetter, E. 2011, PhRvD, 83, 064008

Rosswog, S., \& Liebendörfer, M. 2003, MNRAS, 342, 673

Rosswog, S., Ramirez-Ruiz, E., \& Davies, M. B. 2003, MNRAS, 345, 1077

Röver, C., Bizouard, M., Christensen, N., et al. 2009, PhRvD, 80, 102004

Ruffert, M., Janka, H.-T., \& Schäfer, G. 1996, A\&A, 311, 532

Scheck, L., Janka, H.-T., Foglizzo, T., \& Kifonidis, K. 2008, A\&A, 477,931

Scheck, L., Kifonidis, K., Janka, H.-T., \& Müller, E. 2006, A\&A, 457, 963
Scheidegger, S., Whitehouse, S. C., Käppeli, R., \& Liebendörfer, M. 2010 CQGra, 27, 114101

Schnetter, E., Hawley, S., \& Hawke, I. 2004, CQGra, 21, 1465

Shibata, M., \& Nakamura, T. 1995, PhRvD, 52, 5428

Shoemaker, D. 2010, Advanced LIGO Anticipated Sensitivity Curves, Tech. Rep. LIGO-T0900288-v3, LIGO Scientific Collaboration

Somiya, K. (for the KAGRA Collaboration) 2012, CQGra, 29, 124007

Steiner, A. W., Lattimer, J. M., \& Brown, E. F. 2010, ApJ, 722, 33

Sumiyoshi, K., \& Yamada, S. 2012, ApJS, 199, 17

Sumiyoshi, K., Yamada, S., Suzuki, H., et al. 2005, ApJ, 629, 922

Takiwaki, T., Kotake, K., \& Suwa, Y. 2012, ApJ, 749, 98

Thompson, T. A., Burrows, A., \& Pinto, P. A. 2003, ApJ, 592, 434

Thompson, T. A., Quataert, E., \& Burrows, A. 2005, ApJ, 620, 861

Thornburg, J. 2004, CQGra, 21, 3665

Timmes, F. X., \& Arnett, D. 1999, ApJS, 125, 277

Ugliano, M., Janka, H.-T., Marek, A., \& Arcones, A. 2012, ApJ, 757, 69

Wilson, J. R. 1985, in Numerical Astrophysics, ed. J. M. Centrella, J. M. Leblanc, \& R. L. Bowers (Boston: Jones \& Bartlett), 422

Wongwathanarat, A., Janka, H., \& Müller, E. 2010, ApJL, 725, L106

Woosley, S. E., Heger, A., \& Weaver, T. A. 2002, RvMP, 74, 1015

Yakunin, K. N., Marronetti, P., Mezzacappa, A., et al. 2010, CQGra, 27, 194005

Yamasaki, T., \& Yamada, S. 2007, ApJ, 656, 1019

Zhang, W., Howell, L., Almgren, A., et al. 2013, ApJS, 204, 7 